\title{
Tephrostratigraphy and tephrochronology of lakes Ohrid and Prespa, Balkans
}

\author{
R. Sulpizio ${ }^{1}$, G. Zanchetta ${ }^{2,3}$, M. D'Orazio ${ }^{2}$, H. Vogel ${ }^{4}$, and B. Wagner ${ }^{4}$ \\ ${ }^{1}$ CIRISIVU, c/o Dipartimento Geomineralogico, via Orabona 4, 70125, Bari, Italy \\ ${ }^{2}$ Dipartimento di Scienze della Terra, via S. Maria 53, 56126, Pisa, Italy \\ ${ }^{3}$ INGV sez. Pisa via della Faggiola 4, 56100, Pisa, Italy \\ ${ }^{4}$ Institute for Geology and Mineralogy, University of Cologne, Zülpicher Str. 49A, 50674, Köln, Germany
}

Received: 7 May 2010 - Published in Biogeosciences Discuss.: 28 May 2010

Revised: 21 August 2010 - Accepted: 21 September 2010 - Published: 22 October 2010

\begin{abstract}
Four cores from Balkans lakes Ohrid and Prespa were examined for recognition of tephra layers and cryptotephras, and the results presented along with the review of data from other two already published cores from Lake Ohrid. The six cores provide a previously unrealised tephrostratigraphic framework of the two lakes, and provide a new tephrostratigraphic profile (composite) for the Balkans, which spans from the end of the Middle Pleistocene to the AD 472. A total of 12 tephra layers and cryptotephras were recognised in the cores. One is of Middle Pleistocene age (131 ka) and correlated to the marine tephra layer P-11 from Pantelleria Island. Eight volcanic layers are Upper Pleistocene in age, and encompass the period between ca. $107 \mathrm{ka}$ and ca. $31 \mathrm{ka}$. This interval contains some of the main regional volcanic markers of the central Mediterranean area, including X-6, X-5, Y-5 and Y-3 tephra layers. The other layers of this interval have been related to the marine tephra layers C20, Y-6 and C10, while one was for the first time recognised in distal areas and correlated to the Taurano eruption of probable Vesuvian origin. Three cryptotephras were of Holocene age. Two of which have been correlated to Mercato and AD 472 eruptions of Somma-Vesuvius, while the third has been correlated to the FL eruption from Mount Etna. These recognitions provide a link of the Ohrid and Prespa lacustrine successions to other archives of the central Mediterranean area, like south Adriatic, Ionian, and south Tyrrhenian seas, lakes of southern Italy (Lago Grande di Monticchio, Pantano di San Gregorio Magno and Lago di Pergusa) and Balkans (Lake Shkodra).
\end{abstract}

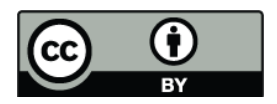

Correspondence to: R. Sulpizio (r.sulpizio@geomin.uniba.it)

\section{Introduction}

Owing to the intense explosive volcanic activity that affected the Mediterranean over the last 200 ky (Vezzoli, 1988; Poli et al., 1987; Santacroce, 1987; Rosi and Sbrana, 1987; Keller et al., 1990; Orsi et al., 1996; Pappalardo et al., 1999; Di Vito et al., 2008; Santacroce et al., 2008) application of tephrochronology to volcanology, Quaternary science, paleoceanography, and archaeology has an exceedingly high potential in this area. In the last 30 years, Quaternary tephra layers have been extensively used to develop a high-resolution event stratigraphy for the late Pleistocene and Holocene across the central and eastern Mediterranean (Keller et al., 1978; Paterne et al., 1988, 1990; Narcisi and Vezzoli, 1999; Wulf et al., 2004; Margari et al., 2007; Aksu et al., 2008; Giaccio et al., 2009; Zanchetta et al., 2010). While early work focused mainly on samples from marine cores (Keller et al., 1978; Paterne et al., 1988, 1990; Calanchi et al., 1998), recent work on terrestrial archives (including Italian, Greek, Turkish and Balkan lakes, and Bulgarian, Greek and Italian cave sites; e.g. St. Seymour and Christianis, 1995; Narcisi and Vezzoli, 1999; St. Seymour et al., 2004; Wulf et al., 2004; Frisia et al., 2008; Giaccio et al., 2008, 2009; Sulpizio et al., 2010; Vogel et al., 2010) have considerably advanced the development of a long, highresolution tephrostratigraphy, which will link marine and terrestrial records of Pleistocene-Holocene-age across the Mediterranean region and mainland Europe. Nevertheless, there are some areas in the Central Mediterranean, in which volcanologic and Quaternary studies are still at an early stage, and the link to the general tephrostratigraphic Mediterranean network is lacking. The Balkans area across Macedonia, Albania and Montenegro is certainly one, in which

Published by Copernicus Publications on behalf of the European Geosciences Union. 
tephrostratigraphic and tephrochronologic studies are in their infancy, although some studies on lacustrine settings indicate the area is extremely promising for tephrostratigraphic studies (Wagner et al., 2008; Caron et al., 2010; Lézine et al., 2010; Sulpizio et al., 2010; Vogel et al., 2010). For these areas both tephrostratigraphic and tephrochronologic studies can offer invaluable stratigraphic support for sedimentologic, palaeoclimatic, paleoenvironmental, and volcanological studies. Here we present tephrostratigraphic and tephrochronologic data of lakes Ohrid and Prespa (Fig. 1), which encompass the last $131 \mathrm{ka}$.

The paper reviews some already published data from the Macedonian side of Lake Ohrid, which are presented along with new data from both Ohrid and Prespa lakes. The recognised tephra layers supply a composite tephrostratigraphy of lakes Ohrid and Prespa, which has been correlated to other archives from the Balkans (Albanian side of Lake Ohrid, Caron et al., 2010; Lézine et al., 2010; Lake Shkodra, Sulpizio et al., 2010), and linked to the regional tephrostratigrapic network of the central Mediterranean area.

\section{Study sites and analytical methods}

\subsection{The lakes of Ohrid and Prespa}

Lake Ohrid (Fig. 1) is a transboundary lake shared by the Republics of Albania and Macedonia. It is located at $693 \mathrm{~m}$ above sea level (a.s.l.), and surrounded by high mountain ranges reaching heights up to $2300 \mathrm{~m}$. It is $30 \mathrm{~km}$ long, $15 \mathrm{~km}$ wide and covers an area of $358 \mathrm{~km}^{2}$. The lake basin shows a relatively simple tub-shaped morphology with a maximum water depth of $289 \mathrm{~m}$, an average water depth of $151 \mathrm{~m}$ and a total volume of $50.7 \mathrm{~km}^{3}$ (Popovska and Bonacci, 2007).

Lake Ohrid is mainly fed by inflow from karst springs $(55 \%)$, while the remaining $45 \%$ of the hydrological input includes direct precipitation on the lake surface, river and direct surface runoff (Matzinger et al., 2006). The direct watershed of Lake Ohrid covers an area of $1002 \mathrm{~km}^{2}$ (Popovska and Bonacci, 2007). Surface outflow (60\%) through the River Crn Drim to the north and evaporation (40\%) are the main hydrological outputs (Matzinger et al., 2006). The average annual precipitation on the Lake Ohrid watershed is $907 \mathrm{~mm}$ (Popovska and Bonacci, 2007).

Lake Prespa is located $10 \mathrm{~km}$ to the east of Lake Ohrid, at an altitude of $849 \mathrm{~m}$ a.s.l. It is a transboundary lake shared between the Republics of Macedonia, Albania, and Greece. The surface area is $254 \mathrm{~km}^{2}$, with a catchment area of ca. $1300 \mathrm{~km}^{2}$, a maximum water depth of $48 \mathrm{~m}$, and a volume of $3.6 \mathrm{~km}^{3}$. The total inflow is estimated to $16.9 \mathrm{~m}^{3} \mathrm{~s}^{-1}$, with $56 \%$ originating from river runoff from numerous small streams, 35\% from direct precipitation, and 9\% from Lake Mikri Prespa to the south (Matzinger et al., 2006). Water loss derives through evaporation (52\%), irrigation (2\%) and outflow through karst aquifers (46\%).

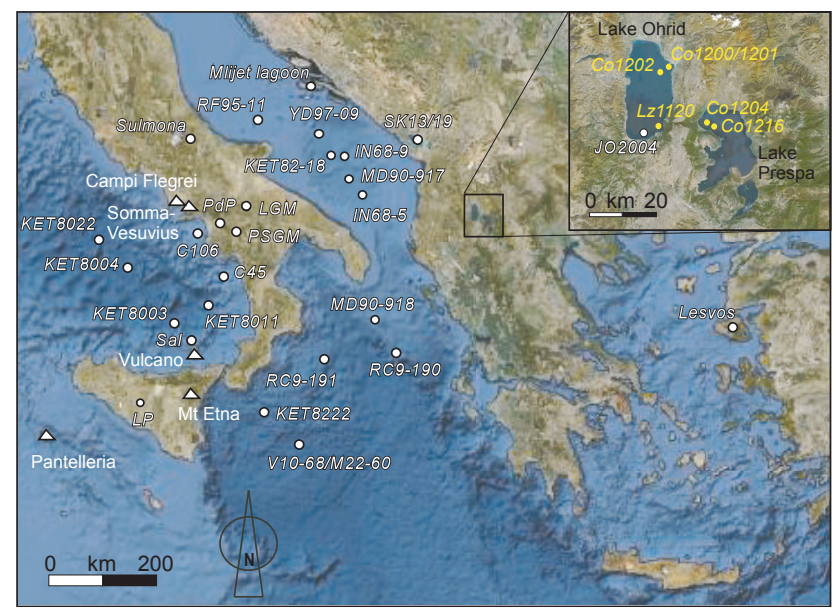

Fig. 1. Location map of the study area. A close view of lakes Ohrid and Prespa is shown in the framework in the upper right angle, along with the locations of the studied cores. Triangles indicate Italian volcanoes of interest for this study. White dots indicate the cores used for tephra correlations: LP, Lago di Pergusa (Sadori and Narcisi, 2001); JO2004, Albanian side of Lake Ohrid (Caron et al., 2010a; Lézine et al., 2010); LGM, Lago Grande di Monticchio (Wulf et al., 2004), Sulmona (Giaccio et al., 2009), YD97-09, RF95-11 (Lowe et al., 2007); IN68-5, IN68-9 (Calanchi and Dinelli, 2008); KET82-18, KET80-03, KET80-04, KET80-011, KET80-22 (Paterne et al., 1988); KET82-22 (Paterne et al., 2008); MD90-917 (Siani et al., 2004); MD90-918 (Caron et al., 2010b; Zanchetta et al., 2010); C45, C146 (Buccheri et al., 2002); V10-68, M22-60, RC9-190, RC9-191 (Keller et al., 1978); PSGM, Pantano San Gregorio Magno (Munno and Petrosino, 2006); Mlijet lagoon (Jahns and van den Boogard, 1998); SK13/19, Shkodra lake (Sulpizio et al., 2010); Lesvos (Margari et al., 2007); PdP, Pian di Pecore (Zanchetta et al., 2010); Sal, Salina island (Lucchi et al., 2008).

\subsection{Core recovery and description}

The sediment cores presented here were recovered during field campaigns between 2005 and 2009, using a floating platform equipped with a gravity corer for surface sediments and a piston corer (both UWITEC Co.) for deeper sediments. Cores Lz1120, Co1200, Co1201, and Co1202 are from Lake Ohrid and cores Co1204 and Co1216 from Lake Prespa (Fig. 1). Core description and high-resolution colour scanning was carried out immediately after lengthwise opening of the cores in the laboratory. Tephrostratigraphy of cores Lz1120 and Co1202 was already published (Wagner et al., 2008; Vogel et al., 2010).

Figure 2 summarises the major lithological features of all cores presented in this study. Detailed lithological descriptions are presented elsewhere (Lz1120, Wagner et al., 2009; Co1200 and Co1201, Lindhorst et al., 2010; Co1202, Vogel et al., 2010a, b; Co1204; Wagner et al., 2010). 


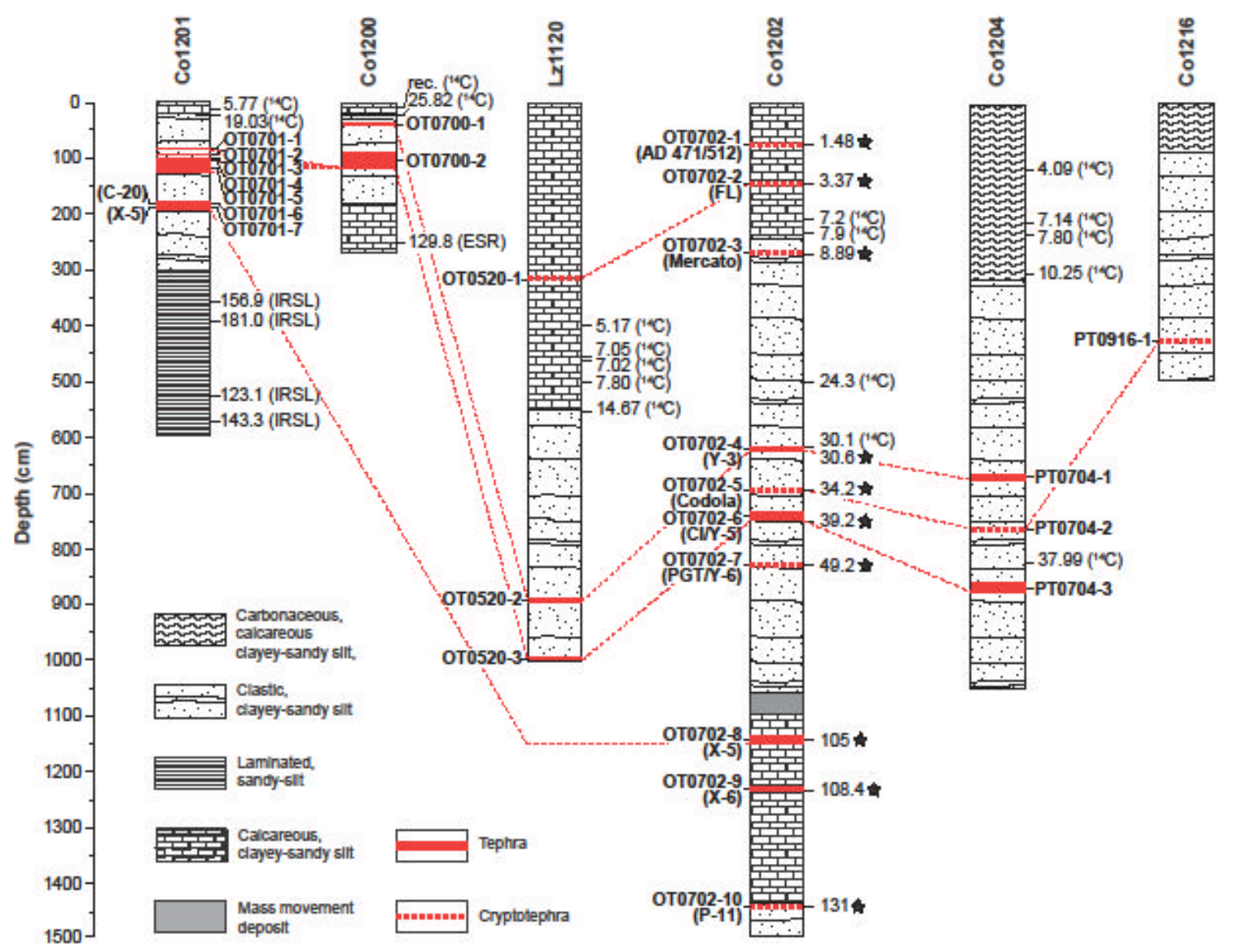

Fig. 2. Summary of stratigraphy and lithology of the studied cores. The thicknesses and sediment depths of tephras and cryptotephras are indicated by red horizons. Nomenclature of tephra layers for cores Co1200 (OT0700-x), Co1201 (OT0701-x), Co1202 (OT0702-x), Lz1120 (OT0520-x), Co1204 (PT0704-x), and Co1216 (PT0916-x) refers to the text. Nomenclature in brackets refers to the coding given to correlated tephra layers and or their parent eruptions. Dashed lines between the cores indicate the correlation of individual tephras and cryptotephras. Independent age control for the different sediment successions is given by radiocarbon (14C) dating for cores Co1200 (Lindhorst et al., 2010), Co1201 (Lindhorst et al., 2010), Co1202 (Vogel et al., 2010a), Lz1120 (Wagner et al., 2008), Co1204 (Wagner et al., 2010) as well as infrared stimulated luminescence (IRSL), and electron spin resonance (ESR) dating for cores Co1200 and Co1201 (Lindhorst et al., 2010 ). Stars indicate literature ages of tephra layers and/or their parent eruptions.

\subsection{Tephra detection and analysis}

High-resolution XRF analysis was carried out on the surface of one of the core halves using an ITRAX core scanner (COX Ltd), equipped with a Mo-tube set to $30 \mathrm{kV}$ and $30 \mathrm{~mA}$, and a Si-drift chamber detector. Scanning was performed at $0.5 \mathrm{~mm}$ (Co1204), $1 \mathrm{~mm}$ (Co1202), $2 \mathrm{~mm}$ (Co1216), and $2.5 \mathrm{~mm}(\mathrm{Co} 1200, \mathrm{Co1201})$ resolution and an analysis time of 20 s (Co1200, Co1201, Co1202, Co1204) and 10 s (Co1216) per measurement. The obtained count rates for K, Sr and $\mathrm{Zr}$ can be used as estimates of the relative concentrations for these elements.

From horizons which were distinctive because of their macroscopic grain size composition, colour or element count rates derived from XRF scanning, about $1 \mathrm{~cm}^{3}$ was washed and sieved. The $>40 \mu \mathrm{m}$ fraction was embedded in epoxy resin and screened for glass shards and micro-pumice frag- ments using scanning electron microscopy (SEM). Energydispersive spectrometry (EDS) of glass shards and micropumice fragments was performed using an EDAX-DX micro-analyser mounted on a Philips SEM 515 (operating conditions: $20 \mathrm{kV}$ acceleration voltage, 100 s live time counting, 200-500 nm beam diameter, 2100-2400 shots s $^{-1}$, ZAF correction). The ZAF correction procedure does not include natural or synthetic standards for reference, and requires analysis normalisation at a given value (chosen at $100 \%$ ). Analytical precision is $0.5 \%$ for abundances higher than $15 \mathrm{wt} \%, 1 \%$ for abundances around $5 \mathrm{wt} \%, 5 \%$ for abundances of $1 \mathrm{wt} \%$, and less than $20 \%$ for abundances close to the detection limit (around $0.5 \mathrm{wt} \%$ ). Interlaboratory standards are shown in Table 1.

Accuracy of measurements is around 1\%, a value analogous to that obtained using wave dispersion spectroscopy (WDS), as tested by Cioni et al. (1998) and Marianelli 
Table 1. Comparison of the EDS device used in this study in comparison with WDS microprobes from GeoForschungsZentrum (GFZ, Potsdam, Germany), and from Saclay (France; Cioni et al., 1998). Reference material comprises volcanic glasses with a chemistry ranging from basalt to trachyte. ALV981R23, CFA47, and KE12 samples from Cioni et al. (1998).

\begin{tabular}{|c|c|c|c|c|c|c|c|c|c|c|c|c|c|c|c|c|c|c|c|c|}
\hline & \multirow{2}{*}{$\begin{array}{r}\text { ALV981R23 } \\
\text { (basalt) } \\
\text { EDS } \\
n=12\end{array}$} & \multicolumn{3}{|c|}{ Saclay } & \multirow{2}{*}{$\begin{array}{r}\text { CFA 47 } \\
\text { (trachyte) } \\
\text { EDS } \\
n=12\end{array}$} & \multicolumn{3}{|c|}{ Saclay } & \multirow{2}{*}{$\begin{array}{r}\text { KE 12 } \\
\text { (pantellerite) } \\
\text { EDS } \\
n=10\end{array}$} & \multicolumn{3}{|c|}{ Saclay } & \multirow{2}{*}{$\begin{array}{r}\text { SMP1-a } \\
\text { (trachyte) } \\
\text { EDS } \\
n=13\end{array}$} & \multicolumn{3}{|c|}{ GFZ } & \multirow{2}{*}{$\begin{array}{r}\text { CA1-a } \\
\text { (trachyte) } \\
\text { EDS } \\
n=18\end{array}$} & \multicolumn{3}{|c|}{ GFZ } \\
\hline & & $s d$ & - & $s d$ & & $s d$ & - & $s d$ & & $s d$ & - & $s d$ & & $s d$ & $n=12$ & $s d$ & & $s d$ & $n=10$ & $s d$ \\
\hline $\mathrm{SiO}_{2}$ & 49.56 & 0.14 & 49.79 & 0.19 & 61.39 & 0.27 & 61.94 & 0.33 & 70.8 & 0.23 & 70.83 & 0.22 & 60.29 & 0.16 & 60.17 & 0.17 & 58.41 & 0.45 & 58.67 & 0.39 \\
\hline $\mathrm{TiO}_{2}$ & 1.30 & 0.07 & 1.28 & 0.05 & 0.48 & 0.09 & 0.42 & 0.05 & 0.31 & 0.06 & 0.28 & 0.02 & 0.48 & 0.07 & 0.46 & 0.03 & 0.53 & 0.09 & 0.48 & 0.01 \\
\hline $\mathrm{Al}_{2} \mathrm{O}_{3}$ & 16.57 & 0.13 & 16.67 & 0.08 & 18.61 & 0.11 & 18.62 & 0.16 & 7.92 & 0.16 & 7.82 & 0.03 & 19.23 & 0.1 & 19.4 & 0.09 & 19.24 & 0.18 & 18.96 & 0.07 \\
\hline $\mathrm{FeO}$ & 8.44 & 0.22 & 8.46 & 0.09 & 2.76 & 0.12 & 2.66 & 0.15 & 8.41 & 0.06 & 8.67 & 0.2 & 3.01 & 0.08 & 3.15 & 0.05 & 3.95 & 0.24 & 4.22 & 0.14 \\
\hline $\mathrm{MnO}$ & 0.22 & 0.03 & 0.14 & 0.06 & 0.24 & 0.13 & 0.18 & 0.04 & 0.38 & 0.07 & 0.29 & 0.03 & 0.29 & 0.13 & 0.26 & 0.04 & 0.18 & 0.09 & 0.14 & 0.03 \\
\hline $\mathrm{MgO}$ & 8.82 & 0.16 & 8.73 & 0.11 & 0.58 & 0.08 & 0.42 & 0.02 & 0.1 & 0.04 & 0 & 0 & 0.22 & 0.06 & 0.36 & 0.02 & 0.99 & 0.15 & 1.01 & 0.1 \\
\hline $\mathrm{CaO}$ & 11.84 & 0.25 & 11.87 & 0.11 & 1.83 & 0.08 & 1.85 & 0.06 & 0.36 & 0.07 & 0.35 & 0.01 & 1.67 & 0.06 & 1.87 & 0.05 & 3.22 & 0.25 & 3.5 & 0.23 \\
\hline $\mathrm{Na}_{2} \mathrm{O}$ & 3.00 & 0.18 & 2.9 & 0.04 & 5.43 & 0.08 & 5.4 & 0.12 & 7.1 & 0.19 & 7.23 & 0.25 & 6.65 & 0.23 & 6.34 & 0.17 & 4.28 & 0.2 & 4.27 & 0.1 \\
\hline $\mathrm{K}_{2} \mathrm{O}$ & 0.1 & 0.05 & 0.05 & 0.01 & 8.14 & 0.04 & 8.02 & 0.16 & 4.29 & 0.07 & 4.19 & 0.15 & 7.23 & 0.21 & 7.1 & 0.08 & 8.6 & 0.29 & 8.21 & 0.26 \\
\hline $\mathrm{P}_{2} \mathrm{O}_{5}$ & - & - & - & - & - & - & - & - & - & - & - & - & 0 & 0 & 0 & 0 & 0 & 0 & 0 & 0 \\
\hline $\mathrm{Cl}$ & - & - & - & - & - & - & - & - & - & - & - & - & 0.92 & 0.05 & 0.9 & 0.02 & 0.6 & 0.06 & 0.55 & 0.03 \\
\hline S & 0.14 & 0.04 & 0.12 & 0 & 0.54 & 0.08 & 0.49 & 0.01 & 0.32 & 0.08 & 0.33 & 0 & - & - & - & - & - & - & - & - \\
\hline
\end{tabular}

and Sbrana (1998). Comparison of EDS and WDS microanalyses carried out on the same samples has shown differences less than $1 \%$ for abundances greater than $0.5 \mathrm{wt} \%$ (e.g. Cioni et al., 1998; Sulpizio et al., 2010) confirming the full comparability of EDS analyses from the Pisa laboratory and data from WDS microprobes.

The concentration of thirty-five trace elements was determined by ICP-MS for four selected samples. About 50$60 \mathrm{mg}$ of bulk sample powders were dissolved in screw-top PFA vessels with a mixture of $\mathrm{HF}$ and $\mathrm{HNO}_{3}$ on a hot-plate at $\sim 120{ }^{\circ} \mathrm{C}$. The sample solutions were then spiked with $\mathrm{Rh}, \mathrm{Re}$ and $\mathrm{Bi}$ as internal standards $\left(20 \mathrm{ng} \mathrm{ml}^{-1}\right.$ in the final solutions) and diluted to $50 \mathrm{ml}$ in polypropylene flasks. Milli-Q purified water $(18.2 \mathrm{M} \Omega \mathrm{cm})$, and $\mathrm{HF}$ and $\mathrm{HNO}_{3}$ Aristar grade were used in each step of sample preparation. Analyses were performed by external calibration using geochemical reference samples as composition- and matrixmatching calibration solutions. The correction procedure includes (i) blank subtraction; (ii) instrumental drift correction using internal standardization and repeated (every 5 samples) analysis of a drift monitor; (iii) oxide-hydroxide interference correction. At the concentration levels of the studied samples, precisions are better than 5\% RSD, except for $\mathrm{Sc}, \mathrm{Ni}$ and $\mathrm{Cu}$ for which the precisions are between 5 and $10 \%$ RSD.

\section{Description of tephra layers}

The studied sediment cores contain a variable number of tephras and cryptotephras, which are described here on the basis of their lithology and composition. Tephra and cryptotephra numbering follows the origin of the core (OT for Ohrid Tephra and PT for Prespa Tephra), the year of core recovery (2005-2009) and the last two identification numbers of the respective core. Glass shards and micropumice fragments from the different cores were compositionally classified using the silica vs. total alkali diagram (TAS; Le Bas et al., 1986; Fig. 3).

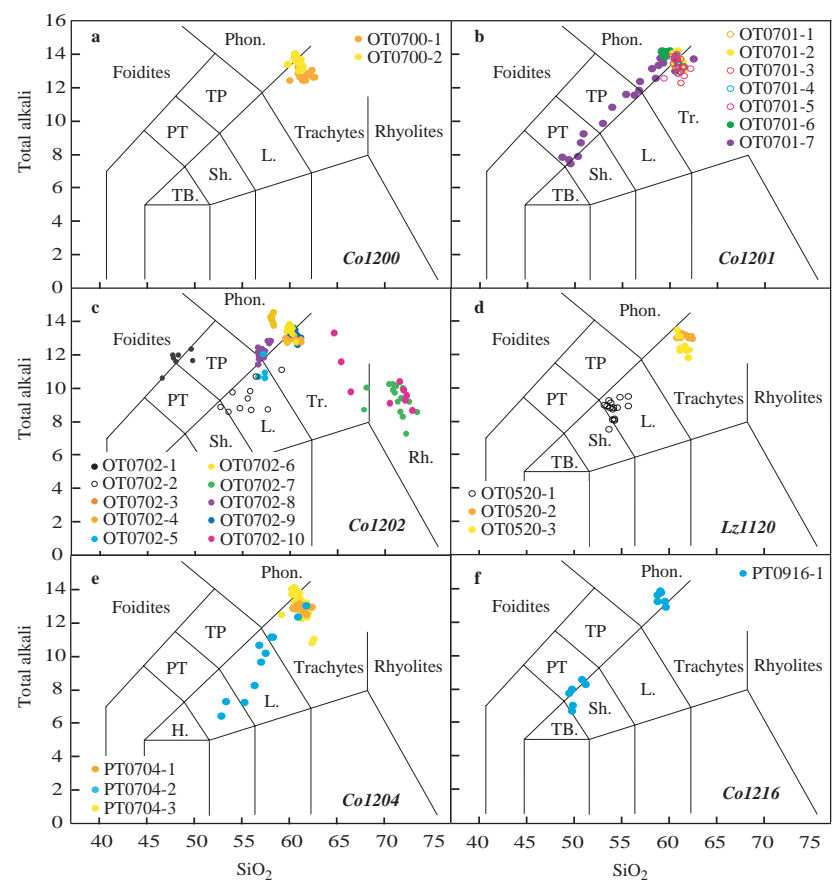

Fig. 3. Total alkali vs. silica diagrams for the tephra layers recognised in the six cores studied or reviewed in this paper.

\subsection{Core Co1200}

Core Co1200 contains two discrete tephra layers visible at naked eye. Tephra layer OT0700-1 (40-38 cm) contains coarse to fine ash, appears light-brown to ochre in colour, and contains highly vesicular, aphyric micro-pumice and glass shards (Fig. 4a). Most of the glass shards show a trachytic composition with only few samples that plot close to the phonolitic field on the TAS diagram (Fig. 3a).

Tephra layer OT0700-2 $(120.5-85.5 \mathrm{~cm})$ comprises coarse to fine ash, has a light red colour, and contains aphyric, 
vesicular micro-pumices and aphyric glass shards with thick septa (Fig. 4b). Glass composition ranges from trachyte to phono-trachyte (Fig. 3a), with three different alkali ratios (Table 2).

\subsection{Core Co1201}

Core Co1201 contains seven discrete tephra layers visible at naked eye inspection and with different thicknesses.

The five tephra layers between $81-78 \mathrm{~cm}$ (OT0701-1), 90-89 $\mathrm{cm}($ OT0701-2), at $100 \mathrm{~cm}$ (OT0701-3), and between 106-104 cm (OT0701-4) and 126-110 cm (OT0701-5) comprise coarse to fine ash, are light red to rusty-red in colour and comprise aphyric micro-pumice and glass shards. They show identical glass composition (Fig. 3b), and are grouped into three different compositional groups on the basis of the different alkali ratios $\left(\mathrm{K}_{2} \mathrm{O} / \mathrm{NaO}\right.$; Table 2).

Tephra layer OT0701-6 $(186-184 \mathrm{~cm})$ comprises coarse to fine ash, appears pink in colour, and contains vesicular, aphyric micro-pumice fragments and glass shards (Fig. 4c). The glass composition is homogeneous phonolitic (Fig. 3b) with alkali ratio greater than 1.5 (Table 2).

Tephra layer OT0701-7 $(192-190 \mathrm{~cm})$ comprises coarse ash, is light-brown to red in colour, and contains both aphyric glass shards and poorly vesicular fragments with small pyroxene crystals in the groundmass (Fig. 4d). The glass composition continuously spans between phonotephryte/shoshonite and phonolite/trachyte (Fig. 3b). Three main compositional groups can be identified on the basis of the alkali ratio and $\mathrm{SiO}_{2}$ content (Table 2).

\subsection{Core Co1202}

Core Co1202 contains four visible tephra layers and six cryptotephras, which were already described in detail by Vogel et al. (2010a).

Cryptotephra OT0702-1 $(77.5-74.5 \mathrm{~cm})$ comprises darkbrown tachylitic fragments with crystalline groundmass (mainly sanidine, clinopyroxene and leucite; Fig. 4e). The composition straddles the foiditic and tephri-phonolitic fields (Fig. 3c).

Cryptotephra OT0702-2 (145.5-144 cm) consists of nonvesicular and blocky fragments with a porphyritic texture. Fragments exhibit mineral inclusions of plagioclase, clinopyroxene and olivine up to some tens of microns in size (Fig. 4f) and frequent occurrences of Fe-Ti oxides. The glass composition is mostly benmoreitic, with few analyses plotting in the trachytic and mugearitic fields on the TAS diagram (Fig. 3c).

Cryptotephra OT0702-3 $(277.5-269 \mathrm{~cm})$ comprises mainly aphyric, vesicular micro-pumice. The bubbles of the micropumices are mainly circular with thick septa (Fig. 4g). The glass composition of the cryptotephra is a fairly homogeneous Na-phonolite (Table 2 and Fig. 3c).

Tephra layer OT0702-4 $(620-617 \mathrm{~cm})$ is light brown in colour, and is characterised by normally graded coarse to fine

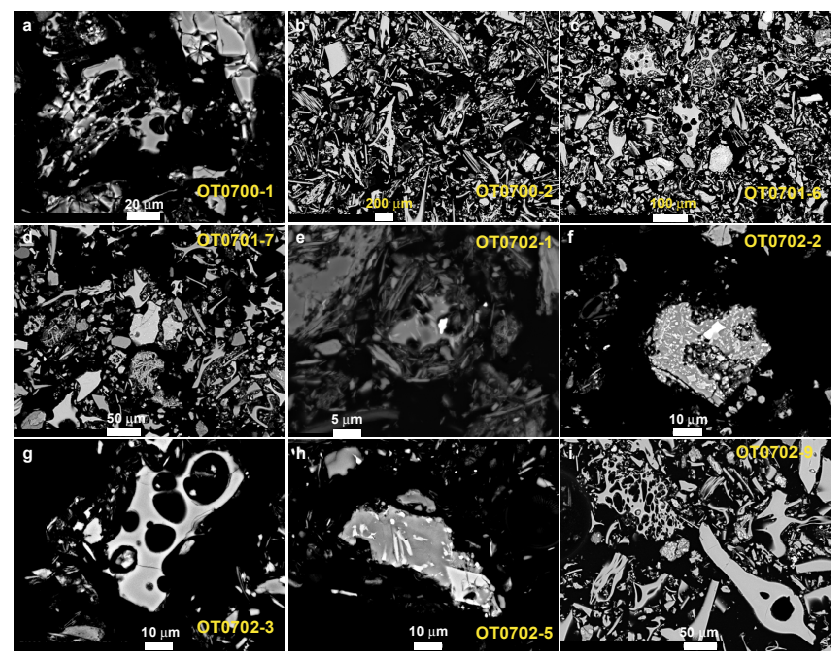

Fig. 4. SEM photographs of volcanic fragments from selected tephra layers and cryptotephras. (a) Y-3 tephra layer in core Co1200; (b) CI/Y-5 tephra layer from core Co1200; (c) C-20 cryptotephra in core Co1201; (d) X-5 tephra layer in core Co1201; (e) AD 472 cryptotephra in core Co1202; (f) FL cryptotephra in Co 1202 core; (g) Mercato cryptotephra in Co1202 core; (h) Codola cryptotephra in core Co1202; (i) X-6 tephra layer core Co1202.

ash. Glass shards are mixed with lacustrine sediment up to $2 \mathrm{~cm}$ above the tephra layer. The tephra comprises highly vesicular, aphyric micro-pumice and cuspate glass shards. Most of the glass shards show a trachytic composition with only few samples that plot close to the phonolitic field on the TAS diagram (Fig. 3c). Trace element distribution (sample 523; Table 3, Fig. 5) shows the less enriched pattern in the four analysed samples, with small negative anomalies in $\mathrm{Ba}$ and $\mathrm{Sr}$ and a marked enrichment in $\mathrm{Pb}$ (Fig. 5a). The rare earth element (REE) pattern shows a more or less regular decrease passing from light REE (LREE) to heavy REE (HREE; Fig. 5b).

Cryptotephra OT0702-5 (696-689 cm) comprises tachilitic particles with a crystal-rich groundmass containing acicular clinopyroxene, plagioclase and sanidine (Fig. 4h). The few glass compositions available range from latite to phonolite when plotted on the TAS diagram (Table 2 and Fig. 3c).

Tephra layer OT0702-6 (752-743 cm) comprises coarse to fine ash, is reddish-brown to light-brown in colour, and glass shards are mixed with lacustrine sediments in the overlying $10 \mathrm{~cm}$. Volcanic particles mainly comprise aphyric, vesicular micro-pumices and aphyric glass shards with thick septa. Glass composition ranges from trachyte to phono-trachyte (Fig. 3c), with two different alkali ratio (Table 2). Trace element distribution (sample 565; Table 3, Fig. 5) shows an intermediate enrichment in the analysed samples, with a pronounced negative anomalies in $\mathrm{Sr}$ and $\mathrm{Eu}$, moderate anomaly in $\mathrm{Ba}$, and moderate positive anomalies in $\mathrm{Th}, \mathrm{U}$ and $\mathrm{Pb}$ (Fig. 5a). The REE pattern shows a regular decrease in 
Table 2. Average EDs analyses of glass shards of tephra layers and cryptotephras from the studued and reviewed cores.

\begin{tabular}{|c|c|c|c|c|c|c|c|c|c|c|c|c|c|c|c|c|c|}
\hline & Sample & depth $(\mathrm{cm})$ & analyses & $\mathrm{SiO}_{2}$ & $\mathrm{TiO}_{2}$ & $\mathrm{Al}_{2} \mathrm{O}_{3}$ & $\mathrm{FeO}_{\text {tot }}$ & $\mathrm{MnO}$ & $\mathrm{MgO}$ & $\mathrm{CaO}$ & $\mathrm{Na}_{2} \mathrm{O}$ & $\mathrm{K}_{2} \mathrm{O}$ & $\mathrm{P}_{2} \mathrm{O}_{5}$ & $\mathrm{ClO}$ & Total & Total alk & Alk. Ratio \\
\hline \multirow[t]{7}{*}{ Co1200 } & OT0700-1 & $40-38$ & $n=12$ & 61.6 & 0.38 & 18.74 & 3.03 & 0.1 & 0.67 & 2.3 & 3.74 & 8.94 & 0 & 0.5 & 100 & 12.68 & 2.39 \\
\hline & & & $s d$ & 0.79 & 0.1 & 0.16 & 0.35 & 0.09 & 0.16 & 0.31 & 0.45 & 0.45 & 0 & 0.14 & & & \\
\hline & ОТ0700-2a & $120.5-85.5$ & $n=11$ & 60.8 & 0.37 & 19.22 & 2.96 & 0.18 & 0.39 & 1.7 & 6.44 & 7.24 & 0 & 0.7 & 100 & 13.69 & 1.13 \\
\hline & & & $s d$ & 0.29 & 0.08 & 0.14 & 0.08 & 0.06 & 0.07 & 0.08 & 0.18 & 0.1 & 0 & 0.07 & & 0.24 & 0.03 \\
\hline & ОТ0700-2b & $120.5-85.5$ & $n=4$ & 61.17 & 0.44 & 19.32 & 2.89 & 0.3 & 0.4 & 1.73 & 5.74 & 7.33 & 0 & 0.69 & 100 & 13.08 & 1.28 \\
\hline & & & sd & 0.16 & 0.13 & 0.13 & 0.07 & 0.04 & 0.05 & 0.07 & 0.09 & 0.16 & 0 & 0.03 & & & \\
\hline & ОТ0700-2c & $120.5-85.5$ & $n=1$ & 59.85 & 0.45 & 18.73 & 3.64 & 0.12 & 0.84 & 2.71 & 3.18 & 10.19 & 0 & 0.3 & 100.01 & 13.37 & 3.2 \\
\hline \multirow[t]{13}{*}{ Co1201 } & OT0701-1/5a & $126-78$ & $n=26$ & 60.8 & 0.42 & 19.15 & 2.99 & 0.23 & 0.39 & 1.68 & 6.38 & 7.26 & 0 & 0.71 & 100 & 13.64 & 1.14 \\
\hline & & & $s d$ & 0.33 & 0.09 & 0.13 & 0.13 & 0.1 & 0.07 & 0.1 & 0.21 & 0.19 & 0 & 0.05 & & 0.27 & 0.05 \\
\hline & OT0701-1/5b & $126-78$ & $n=11$ & 61.32 & 0.4 & 19.13 & 2.99 & 0.14 & 0.48 & 1.87 & 5.53 & 7.55 & 0 & 0.58 & 100 & 13.08 & 1.37 \\
\hline & & & $s d$ & 0.37 & 0.12 & 0.33 & 0.17 & 0.12 & 0.13 & 0.26 & 0.43 & 0.4 & 0 & 0.18 & & & \\
\hline & OT0701-1/5c & $126-78$ & $n=4$ & 60.53 & 0.36 & 19.19 & 3.24 & 0.09 & 0.71 & 2.43 & 3.44 & 9.69 & 0 & 0.33 & 99.99 & 13.13 & 2.83 \\
\hline & & & $s d$ & 0.82 & 0.09 & 0.83 & 0.22 & 0.06 & 0.09 & 0.13 & 0.31 & 0.29 & 0 & 0.03 & & 0.42 & 0.26 \\
\hline & OT0701-6 & $186-184$ & $n=15$ & 59.58 & 0.44 & 20.04 & 2.76 & 0.13 & 0.51 & 2.31 & 5.24 & 8.8 & 0 & 0.18 & 100 & 14.04 & 1.68 \\
\hline & & & $s d$ & 0.23 & 0.07 & 0.12 & 0.14 & 0.09 & 0.09 & 0.09 & 0.16 & 0.17 & 0 & 0.04 & & & \\
\hline & ОТ0701-7a & $192-190$ & $n=8$ & 50.86 & 1.15 & 18.55 & 8.33 & 0.22 & 3.19 & 8.47 & 3.27 & 5.4 & 0.28 & 0.29 & 99.99 & 8.66 & 1.65 \\
\hline & & & $s d$ & 1.79 & 0.16 & 0.33 & 1.26 & 0.06 & 0.59 & 1.15 & 0.45 & 0.78 & 0.14 & 0.04 & & 1.2 & 0.1 \\
\hline & ОТ0701-7b & $192-190$ & $n=13$ & 58.3 & 0.63 & 19.75 & 3.62 & 0.15 & 0.82 & 3.41 & 4.54 & 8.36 & 0.01 & 0.4 & 99.99 & 12.9 & 1.84 \\
\hline & & & $s d$ & 1.48 & 0.12 & 0.2 & 0.89 & 0.08 & 0.43 & 1.07 & 0.31 & 0.62 & 0.03 & 0.05 & & 0.88 & 0.09 \\
\hline & OT0701-7c & $192-190$ & $n=1$ & 62.55 & 0.6 & 18.22 & 2.75 & 0.18 & 0.49 & 0.97 & 7.26 & 6.43 & 0 & 0.56 & 100.01 & 13.69 & 0.89 \\
\hline \multirow[t]{26}{*}{ Co1202 } & OT0702-1 & $77.5-74.5$ & $n=7$ & 48.82 & 0.88 & 20.58 & 7.08 & 0.27 & 1.39 & 8.04 & 6.19 & 5.61 & 0.06 & 1.08 & 100 & 11.8 & 0.92 \\
\hline & & & $s d$ & 1.12 & 0.14 & 0.84 & 0.58 & 0.1 & 0.34 & 1.51 & 0.58 & 0.72 & 0.04 & 0.1 & & & \\
\hline & OT0702-2 & $145.5-144$ & $n=10$ & 55.98 & 1.62 & 18.18 & 6.52 & 0.21 & 1.94 & 5.35 & 5.86 & 3.63 & 0.4 & 0.3 & 100 & 9.49 & 0.62 \\
\hline & $010702-2$ & $175.0-14+4$ & $s d$ & 1.92 & 0.26 & 1.26 & 1.88 & 0.13 & 0.77 & 0.95 & 0.49 & 0.69 & 0.11 & 0.09 & & & \\
\hline & OT0702-3 & $277.5-269$ & $n=9$ & 59.1 & 0.17 & 21.58 & 1.95 & 0.17 & 0.16 & 1.76 & 7.56 & 7.02 & 0 & 0.52 & 100 & 14.58 & 0.93 \\
\hline & & & $s d$ & 0.55 & 0.09 & 0.13 & 0.11 & 0.07 & 0.09 & 0.13 & 0.56 & 0.27 & 0 & 0.03 & & & \\
\hline & ОT0702-4 & $620-617$ & $n=12$ & 61.27 & 0.39 & 18.7 & 3.11 & 0.11 & 0.67 & 2.35 & 3.73 & 9.22 & 0 & 0.46 & 100 & 12.96 & 2.52 \\
\hline & & & $s d$ & 0.64 & 0.06 & 0.12 & 0.21 & 0.07 & 0.18 & 0.25 & 0.46 & 0.48 & 0 & 0.1 & & & \\
\hline & OT0702-5 & $696-689$ & $n=4$ & 57.54 & 0.79 & 20.54 & $\begin{array}{r}3.7 \\
\end{array}$ & 0.17 & 0.61 & 5.16 & 3.58 & 7.58 & 0 & 0.35 & 100.02 & 11.16 & 2.12 \\
\hline & & & $s d$ & 0.34 & 0.18 & 1.02 & 0.73 & 0.09 & 0.15 & 0.62 & 0.5 & 0.62 & 0 & 0.11 & & & \\
\hline & ОT0702-6a & $752-743$ & $n=10$ & 60.71 & 0.42 & 19.17 & 2.95 & 0.21 & 0.45 & 1.7 & 6.49 & 7.22 & 0 & 0.69 & 100 & 13.71 & 1.11 \\
\hline & & & $s d$ & 0.23 & 0.07 & 0.08 & 0.13 & 0.09 & 0.06 & 0.11 & 0.15 & 0.14 & 0 & 0.04 & & & \\
\hline & ОТ0702-6b & $752-743$ & $n=2$ & 61.27 & 0.31 & 19.01 & 2.89 & 0.13 & 0.58 & 2.05 & 5.5 & 7.81 & 0 & 0.47 & 100 & 13.31 & 1.42 \\
\hline & & & $s d$ & 0.23 & 0.24 & 0.21 & 0.18 & 0.18 & 0.16 & 0.36 & 0.59 & 0 & 0 & 0.14 & & & \\
\hline & ОТ0702-7a & $825-822$ & $n=10$ & 71.89 & 0.45 & 8.31 & 8.07 & 0.34 & 0.09 & 0.34 & 5.24 & 4.36 & 0 & 0.92 & 100 & 9.65 & 0.85 \\
\hline & & & $s d$ & 0.47 & 0.1 & 0.19 & 0.12 & 0.09 & 0.07 & 0.05 & 0.74 & 0.11 & 0 & 0.05 & & & \\
\hline & ОТ0702-7b & $825-822$ & $n=2$ & 68.04 & 0.59 & 11.37 & 7.76 & 0.35 & 0.27 & 0.71 & 5.72 & 4.69 & 0 & 0.52 & 100 & 10.41 & 0.83 \\
\hline & & & $s d$ & 1.64 & 0.11 & 1.25 & 0.18 & 0.08 & 0.1 & 0.16 & 0.71 & 0.16 & 0 & 0.16 & & & \\
\hline & OT0702-8 & $1146.5-1140$ & $n=12$ & 57.6 & 0.55 & 19.49 & 4.48 & 0.16 & 1.22 & 3.77 & 4.18 & 8.09 & 0.01 & 0.46 & 100 & 12.26 & 1.94 \\
\hline & & & $s d$ & 0.48 & 0.08 & 0.16 & 0.34 & 0.07 & 0.16 & 0.33 & 0.19 & 0.45 & 0.03 & 0.05 & & & \\
\hline & OT0702-9 & $1232.5-1229$ & $n=15$ & 61.15 & 0.46 & 18.82 & 3.09 & 0.29 & 0.39 & 1.68 & 6.39 & 7.03 & 0 & 0.71 & 100 & 13.42 & 1.12 \\
\hline & & & $s d$ & 0.31 & 0.07 & 0.12 & 0.14 & 0.09 & 0.11 & 0.08 & 0.64 & 0.52 & 0 & 0.15 & & & \\
\hline & ОТ0702-10a & $1447-1440$ & $n=3$ & 66.18 & 0.43 & 16.23 & 4.14 & 0.23 & 0.2 & 0.84 & 5.85 & 5.79 & 0 & 0.13 & 100.01 & 11.64 & 0.99 \\
\hline & & & $s d$ & 0.89 & 0.16 & 3.11 & 3.16 & 0.2 & 0.21 & 0.12 & 0.79 & 1.39 & 0 & 0.11 & & & \\
\hline & OT0702-10b & $1447-1440$ & $n=6$ & 72.52 & 0.41 & 9.34 & 6.7 & 0.31 & 0.12 & 0.34 & 5.06 & 4.5 & 0 & 0.71 & 100 & 9.55 & 0.91 \\
\hline & & & $s d$ & 0.79 & 0.09 & 1.23 & 0.4 & 0.06 & 0.06 & 0.07 & 0.71 & 0.31 & 0 & 0.08 & & & \\
\hline Lz1120 & OT0520-1 & $315-310$ & $n=15$ & 54.25 & 1.76 & 17.48 & 8.15 & 0 & 2.85 & 6.02 & 5.4 & 3.29 & 0.48 & 0.31 & 100 & 8.69 & 0.61 \\
\hline & & & $s d$ & 0.73 & 0.22 & 0.51 & 0.53 & 0 & 0.33 & 0.5 & 0.41 & 0.34 & 0.1 & 0.06 & & & \\
\hline & ОТ0520-2 & $897-896$ & $n=12$ & 61.88 & 0.29 & 18.63 & 2.92 & 0.03 & 0.5 & 2.2 & 4.18 & 8.82 & 0 & 0.54 & 99.99 & 12.99 & 2.11 \\
\hline & & & $s d$ & 0.52 & 0.08 & 0.19 & 0.23 & 0.06 & 0.15 & 0.19 & 0.47 & 0.51 & 0 & 0.14 & & & \\
\hline & ОТ0520-3a & $1075-1070$ & $n=3$ & 61.16 & 0.43 & 18.96 & 3.03 & 0.23 & 0.35 & 1.72 & 6.17 & 7.08 & 0.05 & 0.81 & 100 & 13.25 & 1.15 \\
\hline & & & $s d$ & 0.15 & 0.02 & 0.07 & 0.09 & 0.01 & 0 & 0.05 & 0.13 & 0.35 & 0.01 & 0.01 & & & \\
\hline & ОТ0520-3b & $1075-1070$ & $n=6$ & 61.94 & 0.43 & 19.09 & 3.03 & 0.2 & 0.46 & 1.97 & 4.79 & 7.39 & 0.07 & 0.65 & 100 & 12.18 & 1.54 \\
\hline & & & $s d$ & 0.38 & 0.04 & 0.2 & 0.07 & 0.05 & 0.13 & 0.25 & 0.43 & 0.56 & 0.04 & 0.19 & & & \\
\hline & ОТ0520-3c & $1075-1070$ & $n=1$ & 61.29 & 0.42 & 19.08 & 3.15 & 0.11 & 0.61 & 2.41 & 3.85 & 8.38 & 0.19 & 0.51 & 100 & 12.24 & 2.18 \\
\hline & & & $s d$ & - & - & - & - & - & - & - & - & - & - & - & & & \\
\hline Co1204 & PT0704-1 & $672.5-667.5$ & $n=10$ & 61.43 & 0.37 & 18.62 & 3.17 & 0.1 & 0.66 & 2.34 & 3.54 & 9.34 & 0 & 0.43 & 100.01 & 12.88 & 2.64 \\
\hline & & & $s d$ & 0.59 & 0.06 & 0.11 & 0.24 & 0.07 & 0.15 & 0.22 & 0.41 & 0.39 & 0 & 0.11 & & & \\
\hline & РТ0704-2a & $767.2-764.2$ & $n=5$ & 55.06 & 0.59 & 22.84 & 3.14 & 0.05 & 1.27 & 9.07 & 3.17 & 4.55 & 0.02 & 0.22 & 99.99 & 7.72 & 1.49 \\
\hline & & & $s d$ & 1.83 & 0.34 & 1.83 & 1.43 & 0.07 & 0.99 & 2.09 & 0.72 & 1.05 & 0.05 & 0.13 & & 1.24 & 0.41 \\
\hline & РТ0704-2b & $767.2-764.2$ & $n=4$ & 57.8 & 0.71 & 21.67 & 3.1 & 0 & 0.61 & 5.06 & 3.28 & 7.45 & 0.09 & 0.24 & 100 & 10.73 & 2.28 \\
\hline & & & $s d$ & 0.64 & 0.11 & 1.68 & 1.25 & 0 & 0.32 & 0.49 & 0.22 & 0.29 & 0.11 & 0.11 & & 0.46 & 0.12 \\
\hline & РT0704-2c & $767.2-764.2$ & $n=2$ & 61.45 & 0.41 & 18.99 & 3.06 & 0.11 & 0.69 & 2.18 & 4.05 & 8.59 & 0 & 0.48 & 100 & 12.64 & 2.32 \\
\hline & & & $s d$ & 0.65 & 0 & 0.07 & 0.35 & 0.16 & 0.35 & 0.62 & 1.48 & 0.98 & 0 & 0.07 & & 0.5 & $\begin{array}{l}2.02 \\
1.09\end{array}$ \\
\hline & РТ0704-3a & $879.3-863.3$ & $n=46$ & 60.77 & 0.43 & 19.13 & 2.97 & 0.25 & 0.41 & 1.66 & 6.39 & 7.29 & 0 & 0.71 & 100 & 13.66 & 4.71 \\
\hline & & & $s d$ & 0.25 & 0.09 & 0.15 & 0.11 & 0.08 & 0.07 & 0.08 & 0.19 & 0.18 & 0.01 & 0.05 & & 0.17 & 5.75 \\
\hline & РТ0704-3b & $879.3-863.3$ & $n=11$ & 61.52 & 0.4 & 19.28 & 2.96 & 0.23 & 0.46 & 1.65 & 5.63 & 7.23 & 0 & 0.64 & 100 & 12.86 & 1.29 \\
\hline & & & $s d$ & 0.55 & 0.1 & 0.43 & 0.18 & 0.09 & 0.09 & 0.09 & 0.24 & 0.42 & 0 & 0.11 & & 0.42 & 0.11 \\
\hline & РТ0704-3c & $879.3-863.3$ & $n=9$ & 60.32 & 0.4 & 18.98 & 3.45 & 0.12 & 0.8 & 2.49 & 3.48 & 9.57 & 0 & 0.39 & & 13.05 & 2.78 \\
\hline & & & $s d$ & 1.01 & 0.12 & 0.12 & 0.46 & 0.09 & 0.19 & 0.4 & 0.36 & 0.37 & 0 & 0.09 & & 0.25 & $\begin{array}{r}2.10 \\
0.4\end{array}$ \\
\hline Co1216 & PT0916-1a & $428.6-425.8$ & $n=6$ & 50.22 & 1.09 & 19.05 & 8.12 & 0.17 & 3.68 & 9.39 & 2.91 & 4.78 & 0.33 & 0.26 & 99.99 & 7.69 & 1.64 \\
\hline & & & $s d$ & 0.7 & 0.14 & 0.42 & 0.49 & 0.09 & 0.33 & 0.74 & 0.29 & 0.49 & 0.11 & 0.06 & & & \\
\hline & РT0916-1b & $428.6-425.8$ & $n=6$ & 59.26 & 0.49 & 20.21 & 2.8 & 0.17 & 0.72 & 2.54 & 4.96 & 8.45 & 0 & 0.42 & 100.01 & 13.41 & 1.7 \\
\hline & & & $s d$ & 0.36 & 0.06 & $\begin{array}{r}0.21 \\
0.19\end{array}$ & 0.18 & 0.04 & 0.07 & 0.15 & 0.25 & 0.19 & 0 & 0.06 & & & \\
\hline
\end{tabular}


Table 3. ICP-MS trace element analyses of four selected samples from lakes Ohrid and Prespa: 523, 565, and 1033 are from core Co1202 (Lake Ohrid), PR628 from core Co1204 (Lake Prespa). Some analyses are reported for comparison and correlation: 896-Oh from core Lz1120 (Di Vito et al., 2008; Zanchetta et al., 2008); JO2004Y3 from core JO2004 (Albanian side of lake Ohrid; Caron et al., 2010; Lézine et al., 2010); TM15 from Lago Grande di Monticchio succession (Wulf et al., 2004; sample courtesy of S. Wulf); SMP1-e from proximal deposits of SMP1-e/Y-3 deposits (Di Vito et al., 2008); JO2004Y5 from core JO2004 (Albanian side of lake Ohrid; Caron et al., 2010; Lézine et al., 2010).

\begin{tabular}{|c|c|c|c|c|c|c|c|c|c|}
\hline $\begin{array}{l}\text { T. layer } \\
\text { sample }\end{array}$ & $\begin{array}{r}\text { OT0702-4 } \\
523\end{array}$ & 896-Oh & JO2004Y3 & TM15 & SMP1-e & $\begin{array}{r}\text { OT0702-6 } \\
565\end{array}$ & $\begin{array}{r}\text { Y5 } \\
\text { PT0704-3 } \\
\text { PR628 }\end{array}$ & JO2004Y5 & $\begin{array}{r}\text { X6 } \\
\text { OT0702-9 } \\
1033\end{array}$ \\
\hline $\mathrm{Li}$ & 37 & 40 & 25 & 36 & 49 & 15 & 16 & 30 & 42 \\
\hline $\mathrm{Be}$ & 8.07 & 8 & 7.72 & 8.6 & 12.2 & 16.17 & 17.33 & 16.96 & 15.36 \\
\hline $\mathrm{Sc}$ & 35 & 7 & 15 & 4 & 4 & 22 & 22 & 10 & 41 \\
\hline V & 44 & 70 & 3 & 52 & 37 & 39 & 46 & 16 & 38 \\
\hline $\mathrm{Cr}$ & 44 & 51 & 28 & 1 & 1 & 24 & 9 & 28 & 37 \\
\hline Co & 32 & 11 & 31 & 3 & 2 & 18 & 11 & 39 & 11 \\
\hline $\mathrm{Ni}$ & 20 & 58 & 8 & 4 & 1 & 39 & 39 & 38 & 38 \\
\hline $\mathrm{Cu}$ & 19 & 11 & 14 & 4 & 4 & 3 & 3 & 31 & 7 \\
\hline $\mathrm{Ga}$ & 16.66 & 0.71 & 35.76 & 0.67 & 18 & 22.76 & 10.45 & 30.86 & 35.58 \\
\hline $\mathrm{Rb}$ & 9 & 246 & 38 & 286 & 321 & 17 & 27 & 33 & 56 \\
\hline $\mathrm{Sr}$ & 46 & 361 & 60 & 534 & 176 & 31 & 16 & 13 & 28 \\
\hline $\mathrm{Y}$ & 11.3 & 1.21 & 37.84 & 1.13 & 36 & 26 & 5 & 17 & 15 \\
\hline $\mathrm{Zr}$ & 44 & 238 & 51 & 264 & 373 & 23 & 59 & 66 & 46 \\
\hline $\mathrm{Nb}$ & 26 & 34 & 27 & 36 & 51 & 26 & 43 & 6 & 36 \\
\hline Cs & 7.83 & 0.59 & 9.13 & 0.63 & 21.3 & 37.16 & 16.08 & 9.93 & 29.78 \\
\hline $\mathrm{Ba}$ & 81 & 962 & 55 & 735 & 76 & 59 & 29 & 27 & 24 \\
\hline $\mathrm{La}$ & 8 & 55 & 44 & 55 & 69 & 32 & 38 & 43 & 23 \\
\hline $\mathrm{Ce}$ & 34 & 106 & 20 & 105 & 134 & 40 & 46 & 10 & 40 \\
\hline $\operatorname{Pr}$ & 41.79 & 12 & 16.67 & 0.46 & 14.6 & 1.22 & 20.89 & 29.34 & 45.87 \\
\hline $\mathrm{Nd}$ & 14 & 42 & 21 & 42 & 51 & 25 & 37 & 15 & 22 \\
\hline $\mathrm{Sm}$ & 3.88 & 0.3 & 8.06 & 0.3 & 9.2 & 3.64 & 37.63 & 45.58 & 34.91 \\
\hline $\mathrm{Eu}$ & 24.08 & 0.1 & 33.87 & 0.1 & 1.69 & 17.09 & 7.55 & 41.91 & 3.78 \\
\hline $\mathrm{Gd}$ & 7.46 & 0.25 & 14.82 & 6 & 6.9 & 25.19 & 39.11 & 10.21 & 23.02 \\
\hline $\mathrm{Tb}$ & 41.88 & 0.07 & 9.16 & 0.06 & 1.08 & 31.3 & 33.26 & 20.91 & 0.67 \\
\hline Dy & 3.35 & 0.21 & 35.41 & 0.17 & 5.8 & 16.94 & 7.16 & 41.62 & 28.53 \\
\hline Ho & 3.57 & 1 & 25.11 & 0.06 & 1.15 & 39.72 & 31.86 & 25.84 & 16.89 \\
\hline $\mathrm{Er}$ & 22.04 & 0.13 & 19.13 & 0.11 & 3.09 & 26.19 & 8.16 & 17.17 & 17.69 \\
\hline $\mathrm{Tm}$ & 2.57 & 0.03 & 11.11 & 0.03 & 0.49 & 44.36 & 15.61 & 18.13 & 2.58 \\
\hline $\mathrm{Yb}$ & 5.86 & 0.12 & 43.91 & 0.11 & 3.11 & 38.2 & 41.77 & 36.09 & 3.64 \\
\hline $\mathrm{Lu}$ & 33.19 & 0.03 & 16.33 & 0.02 & 0.44 & 9.72 & 40.68 & 31.41 & 32.02 \\
\hline $\mathrm{Hf}$ & 13.25 & 0.21 & 13.51 & 6 & 8.2 & 34.44 & 25.95 & 9.9 & 12.99 \\
\hline $\mathrm{Ta}$ & 43.62 & 2 & 37.29 & 0.08 & 2.83 & 11.2 & 3.89 & 30.59 & 21.74 \\
\hline $\mathrm{Tl}$ & 13.92 & 0.09 & 0.66 & 0.11 & 2.35 & 12.18 & 23.61 & 44.45 & 28.97 \\
\hline $\mathrm{Pb}$ & 30 & 39 & 12 & 42 & 51 & 26 & 36 & 44 & 44 \\
\hline $\mathrm{Th}$ & 29.6 & 21.0 & 10.9 & 0.9 & 30.7 & 9 & 3 & 44 & 13 \\
\hline $\mathrm{U}$ & 19.86 & 6 & 44.25 & 0.25 & 0.34 & 33.25 & 42.82 & 19.93 & 39.93 \\
\hline
\end{tabular}

enrichment passing from LREE to HREE, with a pronounced negative Eu anomaly (Fig. 5b).

Cryptotephra OT0702-7 (825-822 cm) comprises aphyric, cuspate glass shards with thin septa and glassy groundmass. Glass composition ranges from rhyolitic (main) to trachytic (Table 2 and Fig. 3c).

A sedimentary hiatus occurs between cryptotephra OT0702-7 and tephra layer OT0702-8, which lasted around 16 kyr (Vogel et al., 2010).
Tephra layer OT0702-8 $(1146.5-1140 \mathrm{~cm})$ is rusty-red in colour and comprises coarse ash and shows sharp basal and top contacts. Volcanic particles are aphyric micro-pumices and glass shards. Glass composition is mainly phonolitic (Table 2 and Fig. 3c).

Tephra layer OT0702-9 $(1232.5-1229 \mathrm{~cm})$ is light-brown in colour, and comprises coarse to fine ash with sharp basal and top boundaries. Grain size mainly comprises fine to coarse ash. Volcanic particles are highly vesicular, aphyric micro-pumices and glass shards with a large variability in 

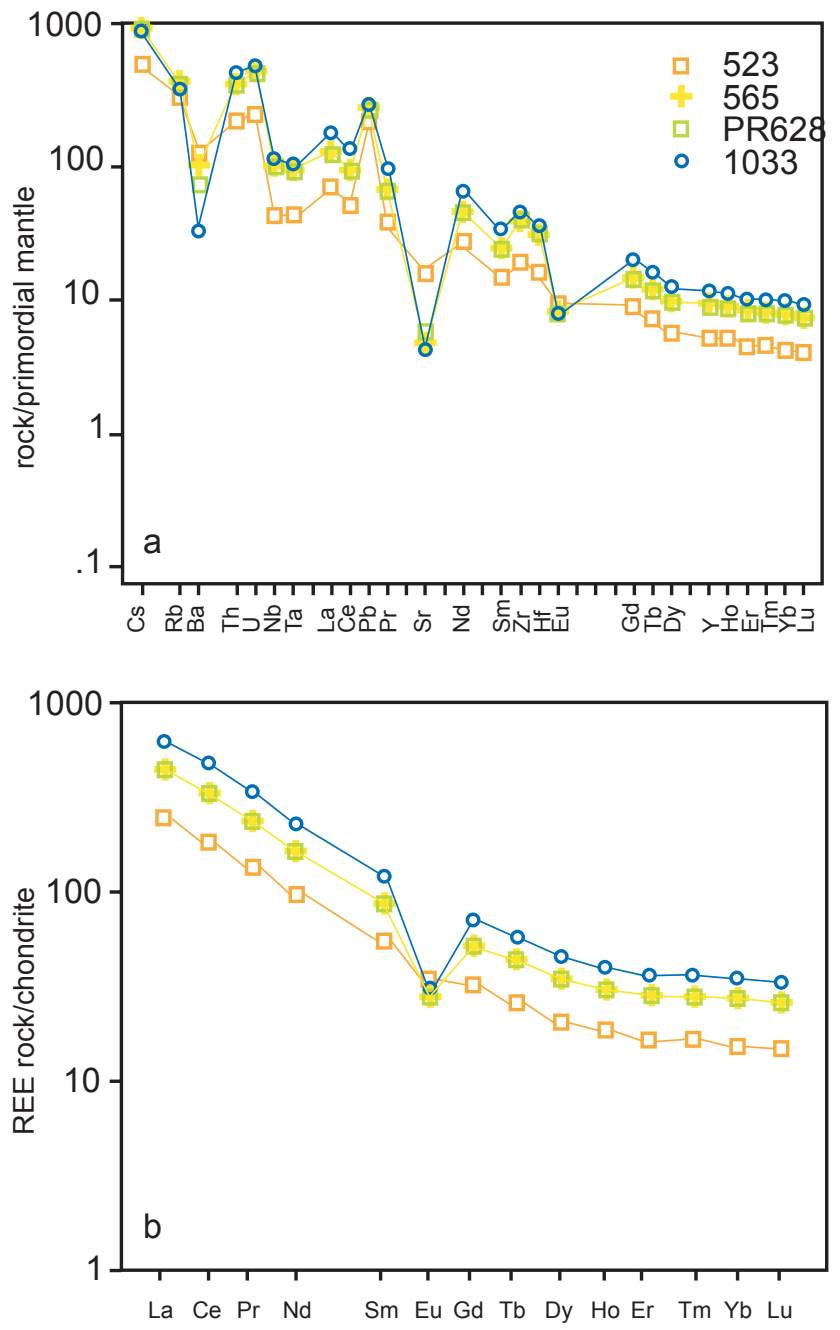

Fig. 5. (a) Primitivemantle-normalised incompatible elements diagram and (b) chondrite-normalised rare earth element (REE) diagram for the selected samples. Samples 523, 535 and 1033 are from core Co1202, the sample PR628 is from core Co1204.

shape and size (Fig. 4i). Glass composition is mainly trachytic, with few analyses that plot into the phonolitic field, and two different alkali ratios (Table 2 and Fig. 3c). The trace element (sample 1003; Fig. 5, Table 3) distribution shows the most enriched pattern in the four analysed samples, with marked negative anomalies in $\mathrm{Ba}, \mathrm{Sr}$ and Eu that testify for a feldspar-dominated magma fractionation (Fig. 5a). The REE pattern shows a regular decrease from LREE to HREE, with a marked negative Eu anomaly (Fig. 5b).

Cryptotephra OT0702-10 (1447-1440 cm) comprises mainly aphyric cuspate glass shards. When plotted on the TAS diagram the glass shards reveal a bimodal chemical composition, which comprises a trachyte and a rhyolite without any compositional trend between (Fig. 3c).

\subsection{Core Lz1120}

Core Lz1120 contains two visible tephra layers and one cryptotephra, which were already described in detail by Wagner et al. (2008).

Cryptotephra OT0520-1 $(315-310 \mathrm{~cm})$ comprises dark brown, blocky fragments with few spherical or ovoid vesicles. The groundmass comprises small crystals of plagioclase and minor olivine. Single-shard analyses show dispersion between mugearitic and benmoreitic fields (Fig. 3d).

Tephra layer OT0520-2 $(897-896 \mathrm{~cm})$ comprises coarse to fine ash, and contains light coloured, elongated, highly vesicular fragments. Vesicles are mainly tubular and form channels throughout the entire length of the pyroclastic fragments. Groundmass is glassy, with very few elongated microcrystals of sanidine. Composition of single shards shows a limited variability within the trachytic field (Fig. 3d).

Tephra OT0520-3 $(1075-1070 \mathrm{~cm})$ is of unknown thickness, as the resistance of this layer prevented penetration of the coring equipment through it. It comprises coarse to fine ash, and volcanic fragments comprise mainly light coloured, highly vesicular fragments, with minor dark coloured glass shards and micropumice fragments. In both typologies of volcanic fragments the vesicles are spherical or ovoidal, separated by thin, glassy sets. Groundmass is almost aphiric, even if small sanidine crystals sometimes occur on larger sets among bubbles. Single shard composition show a trend from the trachytic to the phonolitic fields (Fig. 3d), and can be arranged into three groups on the basis of different alkali ratios (Table 2).

\subsection{Core Co1204}

Core Co1204 was recovered from the north-western part of Lake Prespa (Fig. 1). It contains two discrete tephra layers and one cryptotephra.

Tephra layer PT0704-1 $(672.5-667.5 \mathrm{~cm})$ comprises light grey coarse to fine ash, and contains aphyric, highly vesicular micro-pumice fragments and glass shards with glassy groundmass. The glass composition is homogeneously trachytic (Fig. 3e; Table 2).

Cryptotephra PT0704-2 (767.2-764.2 cm) was identified by high $\mathrm{Sr}$ count rates through high-resolution XRFscanning, and contains tachilitic fragments with highly crystalline groundmass. Glass is rare and shows a compositional trend from shoshonites to trachytes (Fig. 3e). Three different compositional groups were identified on the basis of $\mathrm{SiO}_{2}$, $\mathrm{CaO}$, and total alkali contents (Table 2).

Tephra layer PT0704-3 (879.3-863.3 cm) comprises coarse to fine ash, and contains aphyric, highly vesicular micro-pumice and cuspate to convolute glass shards with glassy groundmass. Glass composition straddles the phonolitic and trachytic fields (Fig. 3e), it can be split into two or three groups depending on the different alkali ratio (Table 2). 
The trace element distribution and the REE pattern (sample PR628; Table 3, Fig. 5) are identical to the sample 565 from core Co1202.

\subsection{Core Co1216}

Core Co1216 war recorevered from the north-western side of Lake Prespa, close to the location of core Co 1204 (Fig. 1). It contains only one cryptotephra, labelled PT0916-1 (Fig. 2). Volcanic fragments comprise tachilitic particles with a crystal-rich groundmass containing acicular clinopyroxene, plagioclase and sanidine, and aphyric glass shards. The glass compositions range from tephri-phonolite/shoshonite to phonolite when plotted on the TAS diagram (Table 2 and Fig. 3f).

\section{Discussion}

\subsection{Correlation to proximal deposits and other distal archives}

The correlation of a distal tephra layer with proximal counterparts is a critical process, which in many cases implies the contemporaneous use of different data, such as glass and mineral composition, chronology, lithology, and stratigraphic position. This is because pyroclastic deposits from the same source, with few exceptions, show closely similar major element composition. Furthermore, pyroclastic deposits from different sources but originating from magmas with similar degree of evolution (e.g. trachytes and rhyolites) are barely distinguishable on the basis of the sole major element composition. In this study, cores Lz1120 and Co1202, which contain correlated tephra layers (Wagner et al., 2008; Vogel et al., 2010), can be used as a reference, particularly because core Co1202 contains the largest number of tephra layers in all studied sediment cores from this region (Fig. 2).

The youngest volcanic deposit was correlated to the AD 472 (1478 cal. yr BP) eruption of Somma-Vesuvius. It occurs as a cryptotephra in core Co1202 (OT0702-1), but has not been recognised in any of the other studied cores (Fig. 2).

The FL ( $3370 \pm 70$ cal. yr BP; Coltelli et al., 2000) cryptotephra occurs in cores Lz1120 (OT0520-1; Fig. 2; Wagner et al., 2008) and Co1202 (OT0702-2; Fig. 2; Vogel et al., 2010) from Lake Ohrid, but has not been found in the successions from Lake Prespa.

The Mercato $(8540 \pm 50$ cal. yr BP; Zanchetta et al., 2010) cryptotephra occurrence is limited to core Co1202 (OT07023; Fig. 2; Vogel et al., 2010) from Lake Ohrid.

Due to its thickness and distinctive, homogeneous trachytic composition (Table 2) the Y-3 tephra layer was recognized in cores Lz1120 (OT0520-2; Wagner et al., 2008), Co1200 (OT0700-1), and Co1202 (OT0702-4; Vogel et al., 2010) from Lake Ohrid and in core Co1204 (PT0704-1) from Lake Prespa (Fig. 2; Table 2). The ICP-MS analyses of trace elements on sample OT0702-4 (Table 3) reinforces the correlation to the proximal deposits of the SMP1-e eruption $(30.67 \pm 0.23$ cal. kyr BP) from the Campi Flegrei caldera, which is indicated as the proximal counterpart of the Y-3 tephra layer (Di Vito et al., 2008; Zanchetta et al., 2008). The occurrence of the Y-3 tephra layer in four out of six cores (Fig. 2) indicates its usefulness as a stratigraphic marker for lakes Ohrid and Prespa.

The Codola ash (inferred age 33 cal. kyr BP; Giaccio et al., 2008 or 34.2 cal. kyr BP; Vogel et al., 2010) occurs as a cryptotephra in core Co1202 (OT0702-5; Fig. 2; Vogel et al., 2010). The Codola fragments have a highly microcrystalline groundmass and a glass composition that straddles the tephri-phonolitic/latitic and the phonolitic/trachytic (Fig. 6a; Di Vito et al., 2008; Giaccio et al., 2008; Santacroce et al., 2008). Based on their stratigraphic position and lithology the tephra layers PT0704-2 in core Co1204 and PT0916-1 in core Co1216 from Lake Prespa are good candidates for correlation to the Codola eruption. The inspection of glass composition illustrates a more complex situation, with some of the analyses from the PT0704-2 and the PT0916-1 samples that plot outside the Codola field (Fig. 6a). In particular, the PT0916-1 analyses define two compositional groups separated by a broad gap in $\mathrm{SiO}_{2}$ and total alkali content (PT0916-1a and PT0916-1b; Fig. 6a; Table 2). Both groups plot outside the Codola field, either in TAS or $\mathrm{SiO}_{2}$ vs. $\mathrm{CaO}$ diagrams (Fig. 6a, b), being more and less evolved, respectively. The less evolved analyses correlate well to the Taurano composition, which defines an evolutionary trend with the Codola samples in the $\mathrm{SiO}_{2}$ vs. $\mathrm{CaO}$ diagram (Fig. 6b). The Taurano eruption was tentatively assigned to the activity of the Somma-Vesuvius volcano, and approximately dated 36-33 cal. kyr BP (Di Vito et al., 2008). Proximal deposits comprise porphyritic dark scoriae of shoshonitic/phono-tephritic composition, with a groundmass rich in microlites of clinopyroxene, sanidine and leucite (Di Vito et al., 2008).

The exact stratigraphic position of Taurano tephra with respect to the Codola deposits in proximal areas is still under debate (Di Vito et al., 2008; Santacroce et al., 2008), because the two deposits have never been described in stratigraphic succession in proximal areas. The tephrostratigraphy of Lago Grande di Monticchio (Wulf et al., 2004) can help in unravelling the stratigraphic position of Taurano deposits. In particular, most of the TM-17 layers (previously attributed to the late activity of Alban Hills volcano; Wulf et al., 2004) show compositional and petrographic similarity with the Taurano deposits (Fig. 6), with glass composition of TM17-d and TM17-e tephra layers that closely matches that of sample PT0916-1a (Fig. 6; Table 4). The TM-17 cluster is splitted into two different groups on the basis of stratigraphic position and glass composition. The first one (TM17c, TM-17d and TM-17e, hereafter Taurano-a) preceeds the deposition the Codola tephra and has a lower total alkali content of the second group (TM17-a and TM17-b, hereafter 
a
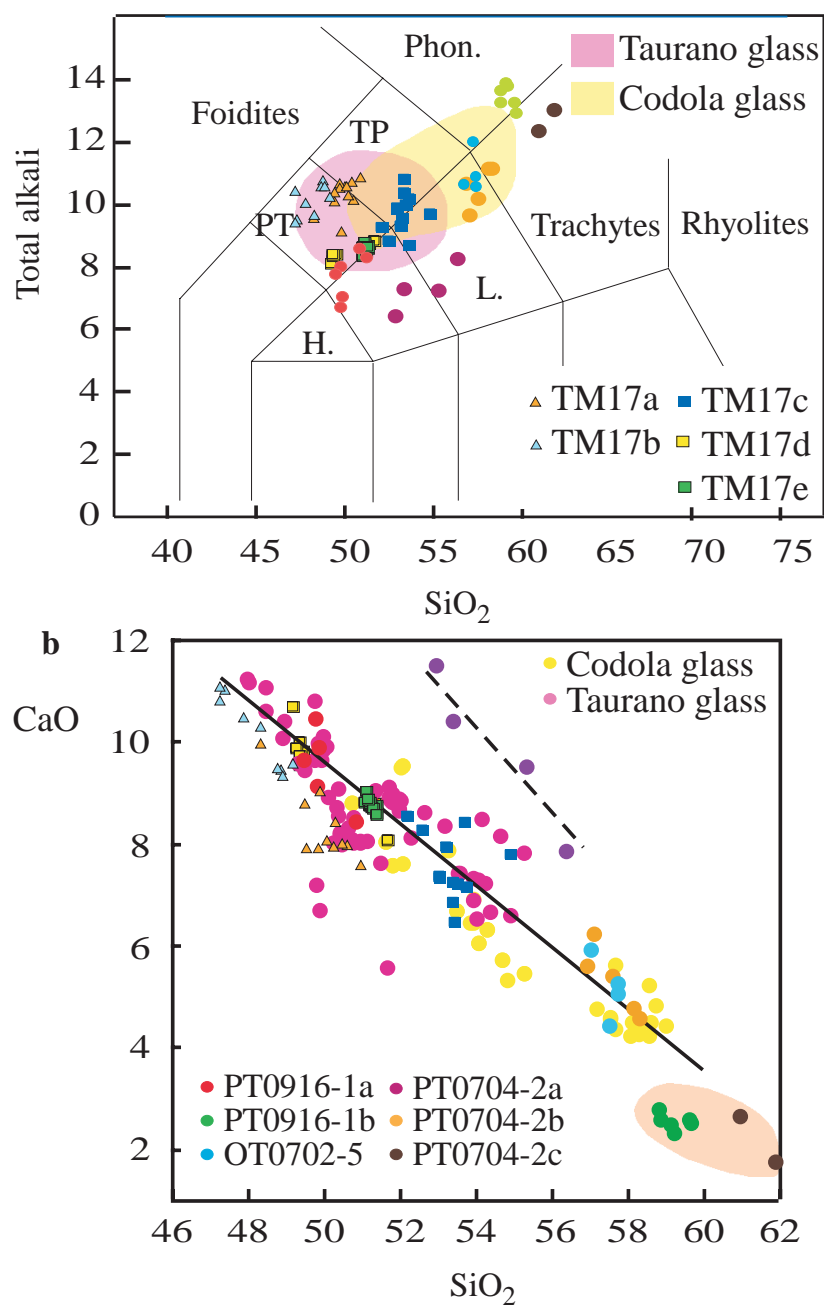

Fig. 6. (a) Total alkali vs. silica diagram for the different groups of OT0702-5 (pale blue dots), PT09-16 (red and green dots), PT07042 (purple, orange and black dots); (b) $\mathrm{CaO}$ vs. $\mathrm{SiO}_{2}$ diagram for the same groups of Fig. 5a. Compositonal fields of proximal deposits of Taurano and Codola eruptions are shown, together with analyses of some TM17 tephra layers from Lago Grande di Monticchio succession (Wulf et al., 2004).

Taurano-b; Fig. 6), which is younger than Codola tephra layer (Wulf et al., 2004). Based on these considerations, the correlation of the tephra OT0916-1a to TM17-d and TM17e tephra layers and to the Taurano-a deposits (age between 34.2 and 36 cal kyr BP) is here proposed.

The evolved group (sample PT0916-1b; Table 2) plots outside the Taurano-Codola trend, and coincides with the Campanian Ignimbrite field in $\mathrm{SiO}_{2}$ vs. $\mathrm{CaO}$ diagram (pinkish area of Fig. 6b). The lithology of the glass shards supports the correlation of this group to the Campanian Ignimbrite deposits, being formed by convolute and cuspate shards with glassy groundmass. The analyses of cryptotephra PT07042 from core Co1204 can be divided into three groups, on the basis of different contents of $\mathrm{SiO}_{2}$, total alkali, and $\mathrm{CaO}$ (Fig. 6a, b; Table 2). Among them, only the PT0704-2b samples plot in the Codola field in both TAS and $\mathrm{SiO}_{2}$ vs. $\mathrm{CaO}$ diagram (Fig. 6a, b). The two analyses of group PT0704-2c are the most evolved, and plot in the Campanian Ignimbrite field in the $\mathrm{SiO}_{2}$ vs. $\mathrm{CaO}$ diagram (Fig. 6b). The PT07042a group have shoshonitic/latitic composition (Fig. 6a), and shows a separate compositional trend in the $\mathrm{SiO}_{2}$ vs. $\mathrm{CaO}$ diagram. The correlation of this group with the regional tephrostratigraphy is at present not possible, since tephra layers or cryptotephras with similar lithology, glass composition and age have never been described in the Balkans, in Adriatic/Ionian marine cores, and continental Italy (e.g. Lago Grande di Monticchio succession; Wulf et al., 2004). Based on glass composition, a generic correlation to the activity of Vulcano Island between 53 and $21 \mathrm{ka}$ (De Astis et al., 2000, 2006; Lucchi et al., 2008) is here proposed, although the link to a specific dated eruption is, as yet, impossible.

The Campanian Ignimbrite/Y-5 (CI/Y-5; $39.28 \pm 0.11 \mathrm{ka}$; de Vivo et al., 2001) tephra layer has previously been recognized in cores Lz1120 (OT0520-3; Fig. 2; Wagner et al., 2008) and Co1202 (OT0702-6; Fig. 2; Vogel et al., 2010a) from Lake Ohrid. The glass composition of the OT0520-3 and the OT0702-6 tephra layers straddles the trachytic and phonolitic fields (Fig. 3c), and can be organised into two to three different compositional groups on the basis of alkali ratios (Table 2). These compositional groups reflect the involvement in the eruptive processes of three differently evolved trachytic magmas (i.e. from "evolved" to "primitive" trachytes; Fedele et al., 2007), which provides a geochemical fingerprint of the CI/Y-5 deposits also in distal reaches (Civetta et al., 1997; Pappalardo et al., 2002). Based on their lithology, stratigraphic position and major element glass composition the tephra layers found in cores Co1200 (OT0700-2; Fig. 2), Co1201 (OT0701-1, -2, -3, -4, -5; Fig. 2) from Lake Ohrid and in core Co1204 (PT0704-3; Fig. 2) from Lake Prespa can also be correlated to OT0520-3 and OT0702-6 and thus to the CI/Y-5 eruption. ICP-MS analyses of trace elements of samples OT0702-6 and PT0704-3 (Table 3; Fig. 5) reinforce this correlation. If we also consider the glass shards mixed into the PT0916-1 cryptotephra, the CI/Y-5 distal ash occurs in all studied cores from lakes Ohrid and Prespa (Fig. 2). The CI/Y-5 tephra layer is the lowermost volcanic deposit in most of the studied cores, with the only exceptions of cores Co1201 and Co1202 (Fig. 2).

Cryptotephra OT0702-7 occurs below the CI/Y-5 tephra layer in core Co1202, and was correlated to the Green Tuff eruption from Pantelleria island (Vogel et al., 2010), which corresponds to the Y-6 marine tephra layer (Keller et al., 1978).

The tephra OT0701-6 occurs below the CI/Y-5 in core Co 1201, and has a homogeneous trachytic composition (Fig. 3b; Table 2). It has no correspondence in the other cores from lakes Ohrid and Prespa, but can be correlated to the C20 marine tephra layer (dated at 79-80 ka; Paterne et 
Table 4. Selected analyses (mainly EDS; WDS, EPMA and only for one case XRF) for tephra layers correlated with those found in core Co1202. Data from Lago Grande di Monticchio are from Wulf et al. (2004). Data from S. Gregorio Magno are from Munno and Petrosino (2007). Data for proximal deposits of Mercato are from Santacroce et al. (2008). For Y-3 data see Zanchetta et al. (2008) and Wagner et al. (2008). For Codola see Di Vito et al. (2008), Giaccio et al. (2008). For CI/Y-5 see Di Vito et al. (2008), Giaccio et al. (2008), and Wagner et al. (2008). For the Y-6 see Margari et al. (2007). For the C-20 see Paterne et al. (1988), for P-11 see Paterne et al. (2008). All the literature analyses were normalised to $100 \%$.

\begin{tabular}{|c|c|c|c|c|c|c|c|c|c|c|c|c|c|c|c|c|}
\hline & & & $\mathrm{SiO}_{2}$ & $\mathrm{TiO}_{2}$ & $\mathrm{Al}_{2} \mathrm{O}_{3}$ & $\mathrm{FeO}_{\text {tot }}$ & $\mathrm{MnO}$ & $\mathrm{MgO}$ & $\mathrm{CaO}$ & $\mathrm{Na}_{2} \mathrm{O}$ & $\mathrm{K}_{2} \mathrm{O}$ & $\mathrm{P}_{2} \mathrm{O}_{5}$ & $\mathrm{ClO}$ & Total & Total alkali & alkali ratio \\
\hline \multirow[t]{8}{*}{ AD 472} & \multirow[t]{2}{*}{ OT0702-1 } & $n=7$ & 48.82 & 0.88 & 20.58 & 7.08 & 0.27 & 1.39 & 8.04 & 6.19 & 5.61 & 0.06 & 1.08 & 100 & 11.8 & 0.91 \\
\hline & & $s d$ & 1.12 & 0.14 & 0.84 & 0.58 & 0.1 & 0.34 & 1.51 & 0.58 & 0.72 & 0.04 & 0.1 & & & \\
\hline & \multirow[t]{2}{*}{ Proximal } & $n=333$ & 49.79 & 0.5 & 22.23 & 4.95 & 0.15 & 1.01 & 5.63 & 9.2 & 5.41 & 0 & 1.12 & 100 & 14.61 & 0.59 \\
\hline & & $s d$ & 1.65 & 0.16 & 0.92 & 1.38 & 0.07 & 0.47 & 1.51 & 2.35 & 2.29 & 0 & 0.53 & & & \\
\hline & \multirow[t]{2}{*}{ Shkodra } & $n=28$ & 50.28 & 0.55 & 22.64 & 4.91 & 0.17 & 0.65 & 5.69 & 8.32 & 5.82 & 0.02 & 0.97 & 100.02 & 14.14 & 0.7 \\
\hline & & $s d$ & 0.83 & 0.11 & 0.62 & 0.53 & 0.07 & 0.29 & 0.67 & 1.02 & 0.92 & 0.03 & 0.1 & & & \\
\hline & \multirow[t]{2}{*}{ LGM/TM2b } & $n=6$ & 50.18 & 0.5 & 21.61 & 4.79 & 0.2 & 0.64 & 5.89 & 7.9 & 6.92 & 0.29 & 1.07 & 99.99 & 14.82 & 0.88 \\
\hline & & $s d$ & 1.02 & 0.07 & 0.19 & 0.24 & 0.04 & 0.2 & 0.61 & 0.35 & 0.72 & 0.17 & 0.19 & & & \\
\hline \multirow[t]{8}{*}{ FL } & \multirow[t]{2}{*}{ ОТ0702-2 } & $n=10$ & 55.98 & 1.62 & 18.18 & 6.52 & 0.21 & 1.94 & 5.35 & 5.86 & 3.63 & 0.4 & 0.3 & 99.99 & 9.49 & 0.62 \\
\hline & & $s d$ & 1.92 & 0.26 & 1.26 & 1.88 & 0.13 & 0.77 & 0.95 & 0.49 & 0.69 & 0.11 & 0.09 & & & \\
\hline & \multirow{2}{*}{ OT0520-1 } & $n=15$ & 54.25 & 1.76 & 17.48 & 8.15 & 0 & 2.85 & 6.02 & 5.4 & 3.29 & 0.48 & 0.31 & 99.99 & 8.69 & 0.61 \\
\hline & & $s d$ & 0.73 & 0.22 & 0.51 & 0.53 & 0 & 0.33 & 0.5 & 0.41 & 0.34 & 0.1 & 0.06 & & & \\
\hline & \multirow[t]{2}{*}{ Shkodra } & $n=14$ & 56.81 & 1.21 & 20.52 & 4.86 & 0.09 & 1.38 & 5.27 & 6.22 & 3.1 & 0.24 & 0.3 & 100 & 9.32 & 0.5 \\
\hline & & $s d$ & 1.78 & 0.44 & 2.39 & 2.49 & 0.09 & 1.27 & 1.83 & 1.04 & 1.26 & 0.22 & 0.11 & & & \\
\hline & \multirow[t]{2}{*}{ Pergusa } & $n=10$ & 53.02 & 1.9 & 17.27 & 8.93 & 0.22 & 2.98 & 5.94 & 5.09 & 3.95 & 0.43 & 0.27 & 100 & 9.04 & 0.78 \\
\hline & & $s d$ & 1.03 & 0.5 & 1.5 & 1.95 & 0.1 & 0.89 & 1.18 & 0.5 & 1.43 & 0.15 & 0.12 & & & \\
\hline \multirow[t]{6}{*}{ Mercato } & \multirow[t]{2}{*}{ ОТ0702-3 } & $n=9$ & 59.1 & 0.17 & 21.58 & 1.95 & 0.17 & 0.16 & 1.76 & 7.56 & 7.02 & 0 & 0.52 & 99.99 & 14.58 & 0.93 \\
\hline & & $s d$ & 0.55 & 0.09 & 0.13 & 0.11 & 0.07 & 0.09 & 0.13 & 0.56 & 0.27 & 0 & 0.03 & & & \\
\hline & \multirow[t]{2}{*}{ Proximal } & $n=40$ & 58.51 & 0.13 & 21.7 & 1.76 & 0.14 & 0.09 & 1.66 & 8.56 & 6.93 & 0 & 0.52 & 100 & 15.49 & 0.81 \\
\hline & & $s d$ & 0.72 & 0.08 & 0.41 & 0.19 & 0.1 & 0.08 & 0.26 & 0.62 & 0.38 & 0 & 0.09 & & & \\
\hline & LGM/TM6b & $n=10$ & 58.68 & 0.14 & 21.41 & 1.8 & 0.18 & 0.07 & 1.76 & 8.58 & 6.76 & 0.02 & 0.58 & 99.98 & 15.34 & 0.79 \\
\hline & & $s d$ & 0.3 & 0.03 & 0.21 & 0.13 & 0.03 & 0.01 & 0.25 & 0.18 & 0.54 & 0.02 & 0.04 & & & \\
\hline SMP1-e/Y-3 & OT0700-1 & $n=12$ & 61.6 & 0.38 & 18.74 & 3.03 & 0.1 & 0.67 & 2.3 & 3.74 & 8.94 & 0 & 0.5 & 100 & 12.68 & 2.39 \\
\hline & & $s d$ & 0.79 & 0.1 & 0.16 & 0.35 & 0.09 & 0.16 & 0.31 & 0.45 & 0.45 & 0 & 0.14 & & & \\
\hline & OT0702-4 & $n=12$ & 61.27 & 0.39 & 18.7 & 3.11 & 0.11 & 0.67 & 2.35 & 3.73 & 9.22 & 0 & 0.46 & 100.01 & 12.95 & 2.47 \\
\hline & & $s d$ & 0.64 & 0.06 & 0.12 & 0.21 & 0.07 & 0.18 & 0.25 & 0.46 & 0.48 & 0 & 0.1 & & & \\
\hline & РT0704-1 & $n=10$ & 61.43 & 0.37 & 18.62 & 3.17 & 0.1 & 0.66 & 2.34 & 3.54 & 9.34 & 0 & 0.43 & 100.01 & 12.88 & 2.64 \\
\hline & & $s d$ & 0.59 & 0.06 & 0.11 & 0.24 & 0.07 & 0.15 & 0.22 & 0.41 & 0.39 & 0 & 0.11 & & & \\
\hline & Proximal & $n=10$ & 62.42 & 0.51 & 18.38 & 2.87 & 0.24 & 0.47 & 1.92 & 4.79 & 8.41 & n.a. & n.a. & 100.01 & 13.2 & 1.76 \\
\hline & & $s d$ & 0.18 & 0.06 & 0.08 & 0.08 & 0.08 & 0.08 & 0.06 & 0.15 & 0.13 & 0 & 0 & & & \\
\hline & LGM/TM15 & $n=19$ & 62.22 & 0.38 & 18.36 & 3.27 & 0.13 & 0.61 & 2.19 & 3.85 & 8.36 & 0.12 & 0.52 & 100.01 & 12.21 & 2.17 \\
\hline & & $s d$ & 0.78 & 0.03 & 0.21 & 0.29 & 0.04 & 0.15 & 0.22 & 0.44 & 0.55 & 0.06 & 0.11 & & & \\
\hline & M25/4-12 & $n=10$ & 61.79 & 0.4 & 18.57 & 3.29 & 0.14 & 0.64 & 2.52 & 3.69 & 8.96 & 0 & 0 & 100 & 12.65 & 2.43 \\
\hline & & $s d$ & 1.21 & 0.05 & 0.22 & 0.32 & 0.09 & 0.19 & 0.4 & 0.44 & 0.95 & 0 & 0 & & & \\
\hline Codola & ОТ0702-5 & $n=4$ & 57.54 & 0.79 & 20.54 & 3.7 & 0.17 & 0.61 & 5.16 & 3.58 & 7.58 & 0 & 0.35 & 100.02 & 11.16 & 2.12 \\
\hline & & $s d$ & 0.34 & 0.18 & 1.02 & 0.73 & 0.09 & 0.15 & 0.62 & 0.5 & 0.62 & 0 & 0.11 & & & \\
\hline & РT0704-2b & $n=5$ & 57.66 & 0.68 & 22.18 & 2.85 & 0 & 0.54 & 5.29 & 3.37 & 7.14 & 0.07 & 0.22 & 100 & 10.5 & 2.12 \\
\hline & & & 0.63 & 0.11 & 1.85 & 1.22 & 0 & 0.31 & 0.66 & 0.28 & 0.75 & 0.11 & 0.11 & & & \\
\hline & Proximal-a & $n=10$ & 53.18 & 0.78 & 18.6 & 7.12 & 0.17 & 1.87 & 6.85 & 3.09 & 7.31 & 0.47 & 0.58 & 100.02 & 10.4 & 2.37 \\
\hline & & $s d$ & 1.04 & 0.04 & 0.82 & 0.51 & 0.03 & 0.56 & 1.54 & 0.29 & 0.63 & 0.04 & 0.18 & & & \\
\hline & Proximal-b & $n=10$ & 57.92 & 0.53 & 19.74 & 3.56 & 0.15 & 0.59 & 4.28 & 3.57 & 9.06 & 0.11 & 0.51 & 100.02 & 12.63 & 2.54 \\
\hline & & $s d$ & 0.07 & 0.03 & 0.13 & 0.17 & 0.01 & 0.05 & 0.06 & 0.05 & 0.14 & 0.02 & 0.05 & & & \\
\hline & LGM/TM16a & $n=11$ & 52.39 & 0.79 & 19.04 & 7.06 & 0.16 & 1.85 & 7.13 & 3.65 & 6.66 & 0.71 & 0.56 & 100 & 10.31 & 1.82 \\
\hline & & $s d$ & 1.69 & 0.09 & 0.86 & 0.6 & 0.03 & 0.31 & 1.24 & 0.43 & 0.62 & 1.05 & 0.11 & & & \\
\hline & LGM/TM16b & $n=6$ & 58.58 & 0.5 & 19.7 & 3.66 & 0.12 & 0.55 & 4.3 & 3.4 & 8.58 & 0.1 & 0.5 & 99.99 & 11.98 & 2.52 \\
\hline & & $s d$ & 0.65 & 0.06 & 0.41 & 0.33 & 0.03 & 0.14 & 0.95 & 0.49 & 0.4 & 0.04 & 0.08 & & & \\
\hline Taurano & PT0916-1a & $n=6$ & 50.22 & 1.09 & 19.05 & 8.12 & 0.17 & 3.68 & 9.39 & 2.91 & 4.78 & 0.33 & 0.26 & 99.99 & 7.69 & 1.64 \\
\hline & & $s d$ & 0.7 & 0.14 & 0.42 & 0.49 & 0.09 & 0.33 & 0.74 & 0.29 & 0.49 & 0.11 & 0.06 & & & \\
\hline & Proximal & $n=72$ & 50.7 & 0.84 & 19.34 & 7.04 & 0.17 & 2.37 & 8.49 & 3.43 & 6.5 & 0.55 & 0.58 & 100 & 9.93 & 1.9 \\
\hline & & $s d$ & 1.7 & 0.12 & 0.94 & 0.81 & 0.04 & 0.95 & 1.14 & 0.85 & 0.89 & 0.3 & 0.15 & & & \\
\hline & LGM/TM17-d & $n=9$ & 50.04 & 0.92 & 18.41 & 7.51 & 0.17 & 3.89 & 9.46 & 2.58 & 5.82 & 0.73 & 0.48 & 100 & 8.4 & 2.25 \\
\hline & & $s d$ & 1.04 & 0.05 & 0.45 & 0.43 & 0.03 & 0.51 & 0.79 & 0.13 & 0.14 & 0.05 & 0.03 & & & \\
\hline & LGM/TM17-e & $n=12$ & 51.26 & 0.86 & 18.84 & 6.96 & 0.15 & 3.42 & 8.75 & 2.62 & 5.93 & 0.73 & 0.48 & 100 & 8.56 & 2.26 \\
\hline & & $s d$ & 0.11 & 0.04 & 0.22 & 0.07 & 0.03 & 0.08 & 0.12 & 0.05 & 0.11 & 0.04 & 0.02 & & & \\
\hline
\end{tabular}


Table 4. Continued.

\begin{tabular}{|c|c|c|c|c|c|c|c|c|c|c|c|c|c|c|c|c|}
\hline & & & $\mathrm{SiO}_{2}$ & $\mathrm{TiO}_{2}$ & $\mathrm{Al}_{2} \mathrm{O}_{3}$ & $\mathrm{FeO}_{\text {tot }}$ & $\mathrm{MnO}$ & $\mathrm{MgO}$ & $\mathrm{CaO}$ & $\mathrm{Na}_{2} \mathrm{O}$ & $\mathrm{K}_{2} \mathrm{O}$ & $\mathrm{P}_{2} \mathrm{O}_{5}$ & $\mathrm{ClO}$ & Total & Total alkali & alkali ratic \\
\hline \multirow[t]{30}{*}{$\mathrm{CI} / \mathrm{Y}-5$} & \multirow[t]{2}{*}{ ОТ0700-2a } & $n=11$ & 60.8 & 0.37 & 19.22 & 2.96 & 0.18 & 0.39 & 1.7 & 6.44 & 7.24 & 0 & 0.7 & 100 & 13.69 & 1.12 \\
\hline & & $s d$ & 0.29 & 0.08 & 0.14 & 0.08 & 0.06 & 0.07 & 0.08 & 0.18 & 0.1 & 0 & 0.07 & & & \\
\hline & \multirow[t]{2}{*}{ ОТ0700-2b } & $n=4$ & 61.17 & 0.44 & 19.32 & 2.89 & 0.3 & 0.4 & 1.73 & 5.74 & 7.33 & 0 & 0.69 & 100 & 13.08 & 1.28 \\
\hline & & $s d$ & 0.16 & 0.13 & 0.13 & 0.07 & 0.04 & 0.05 & 0.07 & 0.09 & 0.16 & 0 & 0.03 & & & \\
\hline & \multirow[t]{2}{*}{ OT0701-1/5a } & $n=36$ & 60.79 & 0.41 & 19.19 & 2.98 & 0.23 & 0.41 & 1.67 & 6.4 & 7.22 & 0 & 0.7 & 100 & 13.62 & 1.13 \\
\hline & & $s d$ & 0.3 & 0.09 & 0.19 & 0.12 & 0.09 & 0.09 & 0.09 & 0.21 & 0.2 & 0 & 0.05 & & & \\
\hline & \multirow[t]{2}{*}{ OT0701-1/5b } & $n=11$ & 61.32 & 0.4 & 19.13 & 2.99 & 0.14 & 0.48 & 1.87 & 5.53 & 7.55 & 0 & 0.58 & 100 & 13.08 & 1.37 \\
\hline & & $s d$ & 0.37 & 0.12 & 0.33 & 0.17 & 0.12 & 0.13 & 0.26 & 0.43 & 0.4 & 0 & 0.18 & & & \\
\hline & \multirow[t]{2}{*}{ ОТ0701-1/5c } & $n=9$ & 60.75 & 0.31 & 18.97 & 3.27 & 0.06 & 0.75 & 2.45 & 3.36 & 9.76 & 0 & 0.32 & 100 & 13.12 & 2.91 \\
\hline & & $s d$ & 0.59 & 0.1 & 0.56 & 0.25 & 0.06 & 0.11 & 0.17 & 0.41 & 0.31 & 0 & 0.07 & & & \\
\hline & \multirow[t]{2}{*}{ ОТ0702-6a } & $n=10$ & 60.71 & 0.42 & 19.17 & 2.95 & 0.21 & 0.45 & 1.7 & 6.49 & 7.22 & 0 & 0.69 & 100 & 13.71 & 1.11 \\
\hline & & $s d$ & 0.23 & 0.07 & 0.08 & 0.13 & 0.09 & 0.06 & 0.11 & 0.15 & 0.14 & 0 & 0.04 & & & \\
\hline & \multirow{2}{*}{ ОТ0702-6b } & $n=2$ & 61.27 & 0.31 & 19.01 & 2.89 & 0.13 & 0.58 & 2.05 & 5.5 & 7.81 & 0 & 0.47 & 100 & 13.31 & 1.42 \\
\hline & & $s d$ & 0.23 & 0.24 & 0.21 & 0.18 & 0.18 & 0.16 & 0.36 & 0.59 & 0 & 0 & 0.14 & & & \\
\hline & ОТ0520-3a & $n=3$ & 61.16 & 0.43 & 18.96 & 3.03 & 0.23 & 0.35 & 1.72 & 6.17 & 7.08 & 0.05 & 0.81 & 100 & 13.25 & 1.15 \\
\hline & & $s d$ & 0.15 & 0.02 & 0.07 & 0.09 & 0.01 & 0 & 0.05 & 0.13 & 0.35 & 0.01 & 0.01 & & & \\
\hline & ОT0520-3b & $n=6$ & 61.94 & 0.43 & 19.09 & 3.03 & 0.2 & 0.46 & 1.97 & 4.79 & 7.39 & 0.07 & 0.65 & 100 & 12.18 & 1.54 \\
\hline & & $s d$ & 0.38 & 0.04 & 0.2 & 0.07 & 0.05 & 0.13 & 0.25 & 0.43 & 0.56 & 0.04 & 0.19 & & & \\
\hline & РT0704-3a & $n=25$ & 60.77 & 0.44 & 19.13 & 2.93 & 0.26 & 0.4 & 1.64 & 6.38 & 7.33 & 0 & 0.71 & 100 & 13.72 & 1.15 \\
\hline & & $s d$ & 0.24 & 0.08 & 0.13 & 0.08 & 0.07 & 0.07 & 0.09 & 0.19 & 0.19 & 0 & 0.05 & & & \\
\hline & РT0704-3b & $n=10$ & 61.34 & 0.43 & 19.45 & 3.06 & 0.17 & 0.59 & 1.85 & 5.13 & 7.47 & 0 & 0.5 & 99.99 & 12.6 & 1.46 \\
\hline & & $s d$ & 0.9 & 0.08 & 0.43 & 0.29 & 0.08 & 0.25 & 0.48 & 0.57 & 0.71 & 0 & 0.14 & & & \\
\hline & РT0704-3c & $n=4$ & 61.22 & 0.41 & 19.08 & 3.27 & 0.11 & 0.79 & 2.2 & 3.26 & 9.31 & 0 & 0.36 & 100 & 12.57 & 2.86 \\
\hline & & $s d$ & 1.02 & 0.08 & 0.17 & 0.29 & 0.13 & 0.17 & 0.36 & 0.46 & 0.93 & 0 & 0.08 & & & \\
\hline & Prox.-PDCs & $n=10$ & 61.9 & 0.4 & 18.35 & 3.09 & 0.21 & 0.47 & 2.03 & 5.15 & 7.74 & 0.09 & 0.59 & 100.02 & 12.89 & 1.5 \\
\hline & & $s d$ & 0.85 & 0.04 & 0.32 & 0.27 & 0.08 & 0.16 & 0.34 & 0.58 & 0.51 & 0.06 & 0.2 & & & \\
\hline & Prox.-Fall & $n=10$ & 61.33 & 0.42 & 19.16 & 2.89 & 0.24 & 0.35 & 1.75 & 5.96 & 7 & 0.05 & 0.84 & 99.99 & 12.96 & 1.17 \\
\hline & & $s d$ & 0.5 & 0.02 & 0.22 & 0.12 & 0.03 & 0.02 & 0.06 & 0.33 & 0.19 & 0.02 & 0.08 & & & \\
\hline & LGM/TM18 & $n=42$ & 61.79 & 0.42 & 19.11 & 2.93 & 0.24 & 0.35 & 1.73 & 5.66 & 6.93 & 0.05 & 0.78 & 99.99 & 12.59 & 1.22 \\
\hline & & $s d$ & 0.46 & 0.02 & 0.21 & 0.09 & 0.02 & 0.02 & 0.07 & 0.71 & 0.29 & 0.03 & 0.05 & & & \\
\hline SA3-a/C-20 & ОT0701-6 & $n=15$ & 59.58 & 0.44 & 20.04 & 2.76 & 0.13 & 0.51 & 2.31 & 5.24 & 8.8 & 0 & 0.18 & 100 & 14.04 & 1.68 \\
\hline & & $s d$ & 0.23 & 0.07 & 0.12 & 0.14 & 0.09 & 0.09 & 0.09 & 0.16 & 0.17 & 0 & 0.04 & & & \\
\hline & Proximal & $n=10$ & 59.71 & 0.51 & 19.41 & 2.97 & 0.25 & 0.5 & 1.98 & 5.26 & 8.8 & - & 0.61 & 100 & 14.06 & 1.67 \\
\hline & & $s d$ & 0.27 & 0.08 & 0.21 & 0.13 & 0.11 & 0.08 & 0.1 & 0.17 & 0.19 & - & 0.03 & & & \\
\hline & C-20 & n.a. & 59.45 & 0.49 & 19.96 & 3.07 & - & 0.32 & 2.73 & 4.4 & 9.51 & - & - & 99.93 & 13.91 & 2.16 \\
\hline TAU1-b/X-5 & OT0702-8 & $n=12$ & 57.6 & 0.55 & 19.49 & 4.48 & 0.16 & 1.22 & 3.77 & 4.18 & 8.09 & 0.01 & 0.46 & 100 & 12.26 & 1.94 \\
\hline & & $s d$ & 0.48 & 0.08 & 0.16 & 0.34 & 0.07 & 0.16 & 0.33 & 0.19 & 0.45 & 0.03 & 0.05 & & & \\
\hline & ОТ0701-7a & $n=8$ & 50.86 & 1.15 & 18.55 & 8.33 & 0.22 & 3.19 & 8.47 & 3.27 & 5.4 & 0.28 & 0.29 & 99.99 & 8.66 & 1.65 \\
\hline & & $s d$ & 1.79 & 0.16 & 0.33 & 1.26 & 0.06 & 0.59 & 1.15 & 0.45 & 0.78 & 0.14 & 0.04 & & & \\
\hline & ОТ0701-7b & $n=8$ & 58.3 & 0.63 & 19.75 & 3.62 & 0.15 & 0.82 & 3.41 & 4.54 & 8.36 & 0.01 & 0.4 & 99.99 & 12.9 & 1.84 \\
\hline & & $s d$ & 1.48 & 0.12 & 0.2 & 0.89 & 0.08 & 0.43 & 1.07 & 0.31 & 0.62 & 0.03 & 0.05 & & & \\
\hline & LGM/TM24a-1 & $n=32$ & 57.23 & 0.51 & 19 & 4.46 & 0.14 & 1.2 & 4.21 & 4.23 & 8.23 & 0.26 & 0.52 & 99.99 & 12.46 & 1.95 \\
\hline & & $s d$ & 0.59 & 0.03 & 0.19 & 0.2 & 0.02 & 0.12 & 0.3 & 0.1 & 0.22 & 0.04 & 0.04 & & & \\
\hline & LGM/TM24a-2 & $n=6$ & 59.48 & 0.41 & 19.14 & 3.6 & 0.14 & 0.73 & 2.94 & 4.26 & 8.62 & 0.13 & 0.55 & 100 & 12.88 & 2.02 \\
\hline & & $s d$ & 0.45 & 0.03 & 0.16 & 0.21 & 0.03 & 0.1 & 0.26 & 0.18 & 0.22 & 0.04 & 0.05 & & & \\
\hline & LGM/TM24b-1 & $n=7$ & 61.25 & 0.38 & 18.86 & 3.22 & 0.14 & 0.6 & 2.51 & 4.1 & 8.32 & 0.1 & 0.51 & 99.99 & 12.42 & 2.03 \\
\hline & & $s d$ & 0.8 & 0.03 & 0.29 & 0.24 & 0.03 & 0.08 & 0.19 & 0.35 & 0.32 & 0.03 & 0.08 & & & \\
\hline & LGM/TM24b-2 & $n=10$ & 58.39 & 0.53 & 18.77 & 4.6 & 0.15 & 1.29 & 3.9 & 3.87 & 7.82 & 0.26 & 0.42 & 100 & 11.69 & 2.02 \\
\hline & & $s d$ & 0.86 & 0.04 & 0.37 & 0.34 & 0.02 & 0.23 & 0.47 & 0.16 & 0.42 & 0.06 & 0.06 & & & \\
\hline$X-6$ & OT0702-9 & $n=15$ & 61.15 & 0.46 & 18.82 & 3.09 & 0.29 & 0.39 & 1.68 & 6.39 & 7.03 & 0 & 0.71 & 100 & 13.42 & 1.12 \\
\hline & & $s d$ & 0.31 & 0.07 & 0.12 & 0.14 & 0.09 & 0.11 & 0.08 & 0.64 & 0.52 & 0 & 0.15 & & & \\
\hline & LGM/TM27-a & $n 06$ & 61.56 & 0.45 & 18.52 & 2.78 & 0.2 & 0.45 & 1.89 & 5.88 & 7.57 & 0.08 & 0.63 & 100.01 & 13.45 & 1.29 \\
\hline & & $s d$ & 0.18 & 0.02 & 0.07 & 0.08 & 0.02 & 0.05 & 0.08 & 0.19 & 0.18 & 0.04 & 0.07 & & & \\
\hline & LGM/TM27-b & $n=10$ & 60.79 & 0.48 & 18.55 & 2.98 & 0.31 & 0.3 & 1.73 & 7.21 & 6.54 & 0.04 & 0.89 & 99.82 & 13.75 & 0.91 \\
\hline & & $s d$ & 0.33 & 0.03 & 0.1 & 0.11 & 0.03 & 0.01 & 0.06 & 0.28 & 0.16 & 0.03 & 0.08 & & & \\
\hline P-11 & OT0702-10a & $n=3$ & 66.18 & 0.43 & 16.23 & 4.14 & 0.23 & 0.2 & 0.84 & 5.85 & 5.79 & 0 & 0.13 & 100.01 & 11.64 & 0.99 \\
\hline & & $s d$ & 0.89 & 0.16 & 3.11 & 3.16 & 0.2 & 0.21 & 0.12 & 0.79 & 1.39 & 0 & 0.11 & & & \\
\hline & OT0702-10b & $n=6$ & 72.52 & 0.41 & 9.34 & 6.7 & 0.31 & 0.12 & 0.34 & 5.06 & 4.5 & 0 & 0.71 & 100 & 9.55 & 0.91 \\
\hline & & $s d$ & 0.79 & 0.09 & 1.23 & 0.4 & 0.06 & 0.06 & 0.07 & 0.71 & 0.31 & 0 & 0.08 & & & \\
\hline & KET82-22 & n.a. & 64.67 & 0.83 & 15.5 & 5.88 & 0 & 0.28 & 1.45 & 6.41 & 4.98 & 0 & 0 & 100 & 11.39 & 0.78 \\
\hline & & $s d$ & 0.68 & 0.1 & 0.71 & 0.19 & 0 & 0.14 & 0.2 & 0.27 & 0.19 & 0 & 0 & & & \\
\hline & Lesvos/ML5-a & $n=10$ & 73.73 & 0.43 & 8.31 & 7.14 & 0.32 & 0.08 & 0.31 & 4.3 & 4.44 & 0.01 & 0.84 & 99.91 & 8.74 & 1.03 \\
\hline & & $s d$ & 0.24 & 0.01 & 0.1 & 0.16 & 0.02 & 0.01 & 0.01 & 0.25 & 0.09 & 0.01 & 0.03 & & & \\
\hline & Lesvos/ML5-b & $n=15$ & 66.23 & 0.75 & 14.32 & 6.06 & 0.3 & 0.27 & 1.16 & 5.59 & 4.99 & 0.1 & 0.17 & 99.94 & 10.58 & 0.89 \\
\hline & & $s d$ & 1.04 & 0.06 & 1.1 & 0.48 & 0.02 & 0.1 & 0.27 & 0.28 & 0.14 & 0.04 & 0.06 & & & \\
\hline
\end{tabular}


al., 1988), and to the proximal deposits of SA3-b eruption from the Campanian area (Table 3).

The tephra layer OT0702-8 occurs in core Co1202 between $825 \mathrm{~cm}$ and $822 \mathrm{~cm}$, and was correlated to the X-5 marine tephra layer $(105 \pm 2 \mathrm{ka}$; Keller et al., 1978; Kraml, 1997), which has been tentatively related to the TAU1b eruption from Campi Flegrei caldera (Di Vito et al., 2008). Due to its chemical composition the OT0701-3 cryptotephra from core Co1201 can tentatively be correlated to the OT0702-8 tephra layer from core Co1202 and thus to the TAU1-b/X-5 eruption.

Tephra layer OT0702-9 in core Co1202 was correlated to the marine X-6 tephra layer (Keller et al., 1978), of generic Campanian origin. Brauer et al. (2007) quoted an ${ }^{40} \mathrm{Ar} /{ }^{39} \mathrm{Ar}$ age of $107 \pm 2 \mathrm{ka}$ for the X-6 tephra, which is in good agreement with the suggested age of $108.43 \mathrm{ka}$ obtained from the varve-supported chronology of the Lago Grande di Monticchio record. The X-6 tephra layer has not been recognised in the other studied cores of lakes Ohrid and Prespa (Fig. 2). The evolved trace element composition of this tephra layer (Fig. 5; Table 3) can help in its discrimination from the mess of trachytic tephra layers recognised in the Upper Pleistocene successions.

Cryptotephra OT0702-10 (Fig. 2; Vogel et al., 2010a) in core Co1202 from Lake Ohrid represents the deepest volcanic deposit recognised in cores from lakes Ohrid and Prespa. It correlates to the marine P11 tephra layer (ca. 131 ka; Paterne et al., 1988, 2008), which sourced from Pantelleria Island.

\subsection{Composite tephrochronological record and regional correlations}

The correlation of the 12 recognised tephra and cryptotephra layers with known proximal deposits allows the reconstruction of a composite stratigraphic and chronological framework for lakes Ohrid and Prespa during the past $131 \mathrm{kyr}$ (Fig. 7). Three cryptotephras occur during Holocene, with the Mercato layer marking the temperature maximum of the Early Holocene. The following seven tephra layers and cryptotephras punctuate the Upper Pleistocene, encompassing about $77 \mathrm{kyr}$ (from 30 to $107 \mathrm{ka}$; Fig. 7). Four markers cluster between 30 and $40 \mathrm{ka}$ (i.e. between the Y-3 and the Y-5 tephra layers), detailing the stratigraphic succession between Heinrich events 3 and 4 (Ton-That et al., 2001; Zanchetta et al., 2008; Wagner et al., 2010). Particularly significant is the first recognition of the Taurano deposits in a very distal succession, which so far has never been described in nearby Adriatic and Ionian sea cores. The Y-6 cryptotephra occurs below the CI, at about $49 \mathrm{ka}$ (Fig. 7), linking the Balkans to the Ionian sea archives. The sedimentary record below the Y6 spans about $30 \mathrm{kyr}$ without occurrence of any tephra record, being the following tephra layer correlated to the SA3-a eruption and the C-20 marine tephra layer (79-80 ka; Paterne et al., 1988; Fig. 7). The last two tephra layers of the Upper

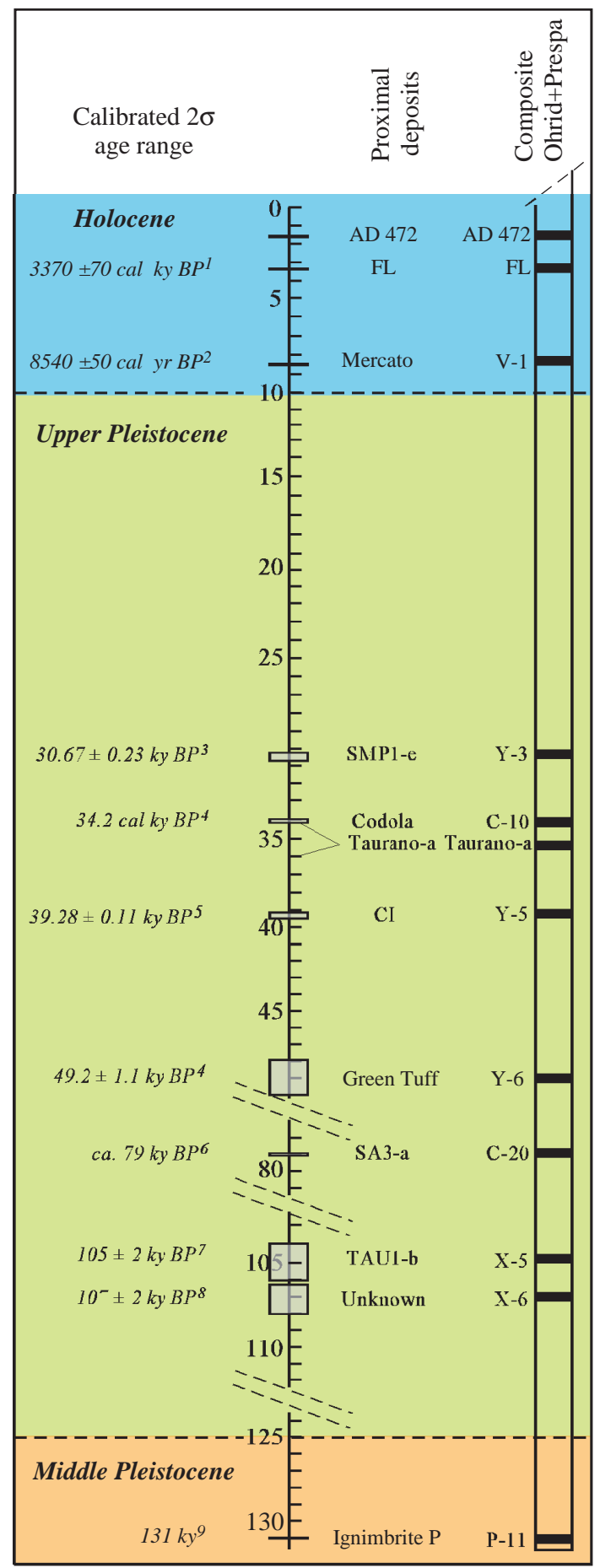

Fig. 7. Updated tephrochronology for the last $131 \mathrm{kyr}$ of lakes Ohrid and Prespa. Grey boxes indicate the age uncertainties for calibrated ${ }^{14} \mathrm{C}$ data and for ${ }^{39} \mathrm{Ar} /{ }^{40} \mathrm{Ar}$ ages. ${ }^{1}$ Coltelli et al. (2000); ${ }^{2}$ Zanchetta et al. (2010); ${ }^{3}$ Di Vito et al. (2008); ${ }^{4}$ Vogel et al. (2010); ${ }^{5}$ De Vivo et al. (2001); ${ }^{6}$ Paterne et al. (1988); ${ }^{7}$ Kraml (1997); ${ }^{8}$ Allen et al. (1999); ${ }^{9}$ Paterne et al. (2008). The three colours indicate the Middle Pleistocene, Upper Pleistocene and Holocene. 


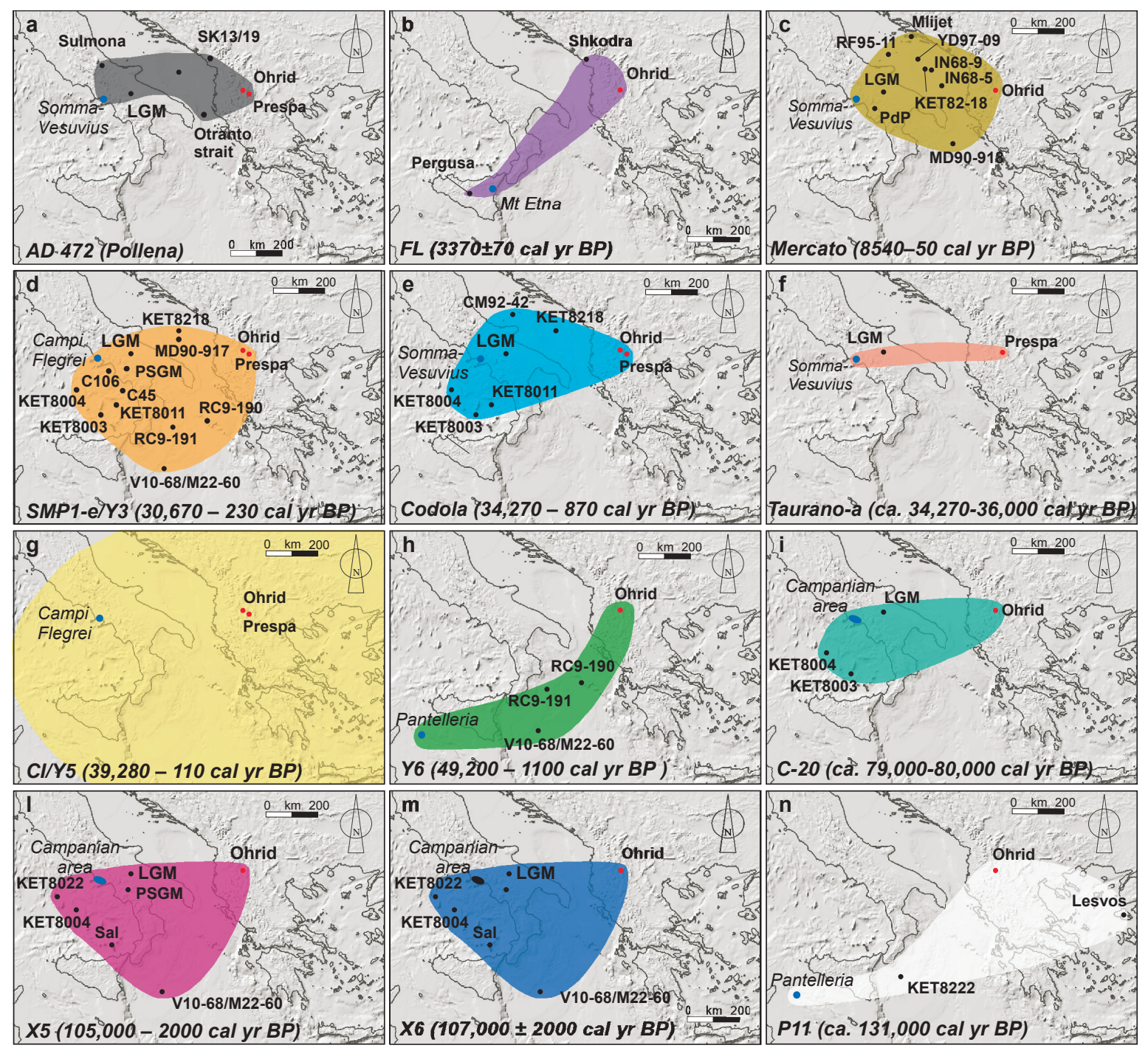

Fig. 8. Dispersal maps of the twelve tephra layers recognised in lakes Ohrid and Prespa.

Pleistocene are the X-5 and X-6, dated at 105 and $107 \mathrm{ka}$, respectively (Fig. 7). The oldest and deepest volcanic deposit recognised is the P-11 tephra layer, which precedes in date the inception of the Last Interglacial in the Balkans (Lézine et al., 2010; Vogel et al., 2010b).

The recognised tephra layers and cryptotephras can be correlated to other published tephrostratigraphic records of the central Mediterranean area, linking the Balkans with other archives at a regional scale (Fig. 8). Excluding the CI/Y-5 tephra layer, which extensively occurs in the central Mediterranean area and extends through Aegean Sea and up to Russia (Pyle et al., 2006; Giaccio et al., 2008), the other recognised volcanic markers have various frequency of occurrence in the published archives (Fig. 8). The most common is the Y-3 tephra layer, which commonly occur in marine cores from south Adriatic (SA), Ionian, and south Tyrrhenian (ST) seas, and in Lago Grande di Monticchio succession (Fig. 8).
Other widespread layers are the Mercato, X-5, and X-6, while the AD 472, and FL deposits link the Balkans to the Monticchio succession, to the Campanian area, and to the Sicily (Fig. 8). The P-11 cryptotephra is the only, together with the Y-5 tephra layer, that links the Balkans to the Ionian Sea and to the Aegean Sea. This is because the pantelleritic/trachytic deposit recognised in a core from Lesvos Island, and attributed to the Pantelleria Green Tuff/Y-6 (Margari et al., 2007), was reinterpreted as the P-11 deposit on the basis of geochemical data (Vogel et al., 2010).

\section{Supplementary material related to this article is available online at: http://www.biogeosciences.net/7/3273/2010/ bg-7-3273-2010-supplement.zip.}


Acknowledgements. Samples from Lago Grande di Monticchio core are a courtesy of Sabine Wulf. RS and GZ thanks INGV-DPC projects for supporting studies on distal ash layers. Biagio Giaccio, Anthony Newton, Massimo Pompilio and David Pyle are greatly acknowledged for the revision of the manuscript.

Edited by: T. Wilke

\section{References}

Aksu, A. E., Jenner, G., Hiscott, R. N., and Isler, E. B.: Occurrence, stratigraphy and geochemistry of Late Quaternary tephra layers in the Aegean Sea and the Marmara Sea, Mar. Geol., 252, 174$192,2008$.

Brauer, A., Allen, J. R. M., Mingram, J., Dulski, P., Wulf, S., and Huntley, B.: Evidence for last interglacial chronology and environmental change from Southern Europe, Proc. Nat. Acad. Sc., 104, 450-455, 2007.

Buccheri, G., Capretto, G., Di Donato, V., Esposito, P., Ferruzza, G., Pescatore, T., Russo Ermolli, E., Senatore, M. R., Sprovieri, M., Bertoldo, M., Carella, D., and Madonia, G.: A high resolution record of the last deglaciation in the southern Tyrrhenian Sea: environmental and climatic evolution, Mar. Geol., 186, 447-470, 2002.

Calanchi, N. and Dinelli, E.: Tephrostratigraphy for the last $170 \mathrm{ka}$ in sedimentary successions from the Adriatic sea, J. Volcanol. Geoth. Res., 177, 81-95, 2008.

Calanchi, N., Cattaneo, A., Dinelli, E., Gasparotto, G., and Lucchini, F.: Tephra layers in Late Quaternary sediments of the Central Adriatic Sea, Mar. Geol., 149, 191-209, 1998.

Caron, B., Sulpizio, R., Zanchetta, G., Siani, G., and Santacroce, R.: The Late Holocene to Pleistocene tephrostratigraphic record of Lake Ohrid (Albania), Compt. Rend. Acad. Sc., 342, 453-466, 2010.

Cioni, R., Marianelli, P., and Santacroce, R.: Thermal and compositional evolution of the shallow magma chambers of Vesuvius: evidence from pyroxene phenocrysts and melt inclusions, J. Geophys. Res., 103, 18277-18294, 1998.

Civetta, L., Orsi, G., Pappalardo, L., Fisher, R. V., Heiken, G., and Ort, M.: Geochemical zoning, mingling, eruptive dynamics and depositional processes - the Campanian Ignimbrite, Campi Flegrei caldera, Italy, J. Volcanol. Geoth. Res., 75, 183-219, 1997.

Coltelli, M., Del Carlo, P., and Vezzoli, L.: Stratigraphic constraints for explosive activity in the past $100 \mathrm{ka}$ at Etna volcano, Italy, Int. J. Earth Sci., 89, 665-677, 2000.

De Astis, G., Peccerillo, A., Kempton, P. D., La Volpe, L., and Tsai $\mathrm{Wu}, \mathrm{W}$.: Transition from calc-alkaline to potassium-rich magmatism in subduction environments: geochemical and $\mathrm{Sr}, \mathrm{Nd}$, $\mathrm{Pb}$ isotopic constraints from the island of Vulcano (Aeolian arc), Contrib. Mineral. Petr., 139, 684-703, 2000.

De Astis, G., Dellino, P., La Volpe, L., Lucchi, F., and Tranne, C. A.: Geological map of the island of Vulcano (Aeolian Islands), University of Bari, University of Bologna, and INGV; printed by L.A.C., Firenze, 2006.

De Vivo, B., Rolandi, G., Gans, P. B., Calvert, A., Bohrson, W. A., Spera, F. J., and Belkin, H. E.: New constraints on the pyroclastic eruptive history of the Campanian volcanic Plain (Italy), Min. Petrol., 73, 47-65, 2001.
Di Vito, M., Sulpizio, R., Zanchetta, R., and D'Orazio, M.: The late Pleistocene pyroclastic deposits of the Campanian Plain: new insights on the explosive activity of Neapolitan volcanoes, J. Volcanol. Goeth. Res., 177, 19-48, 2008.

Fedele, L., Scarpati, C., Lanphere, M., Melluso, L., Morra, V., Perrotta, A., and Ricci, G.: The Breccia Museo formation, Campi Flegrei, southern Italy: geochronology, chemostratigraphy and relationship with the Campanian Ignimbrite eruption, Bull. Volcanol., 70, 1189-1219, 2007.

Frisia, S., Borsato, A., and Susini, J.: Synchrotron radiation applications to past volcanism archived in speleothems: An overview, J. Volcanol. Geoth. Res., 177, 96-100, 2008.

Giaccio, B., Isaia, R., Fedele, F. G., Di Canzio, E., Hoffecker, J., Ronchitelli, A., Sinitsyn, A., Anikovich, M., and Lisitsyn, S. N.: The Campanian Ignimbrite and Codola tephra layers: two temporal/stratigraphic markers for the Early Upper Palaeloithic in southern Italy and eastern Europe, J. Volcanol. Geoth. Res., 177, 208-226, 2008.

Giaccio, B., Messina, P., Sposato, A., Voltaggio, M., Zanchetta, G., Galadini, F., Gori, S., and Santacroce, R.: Tephra layers from Holocene lake sediments of the Sulmona basin, central Italy: implications for volcanic activity in Peninsular Italy and tephrostratigraphy in the central Mediterranean area, Quat. Sci. Rev., 28, 2710-2733, 2009.

Jahns, S. and van den Boogard, C.: New palynological and tephrostratigraphical investigations of two salt lagoons on the island of Mljet, south Dalmatia, Croatia, Veg. Hist. Archaeobot., 7, 219234, 1998.

Keller, J., Ryan, W. B. F., Ninkovich, D., and Altherr, R.: Explosive volcanic activity in the Mediterranean over the past 200,000 years as recorded in deep-sea sediments, Geol. Soc. Am. Bull., 89, 591-604, 1978.

Keller, J., Rehren, T., and Stadkbauer, E.: Explosive volcanism in the Hellenic Arc: a summary and review, in: Thera and the Aegean World III, edited by: Hardy, D. A., Keller, J., Galanopoulos, V. P., Flemming, N. C., and Druitt, T. H., Thera Foundation: Santorini, Greece, 13-26, 1990.

Kraml, M.: Laser-40Ar/39Ar-Datierungen an distalen marinen tephren des jung-quartären mediterranen Vulkanismus (Ionisches Meer, METEOR-Fahrt 25/4), Ph.D. thesis, Albert-LudwigsUniversität Freiburg i.Br., 216 pp., 1997.

Le Bas, M. J., Le Maitre, R. W., Streckeisen, A., and Zanettin, B.: A chemical classification of volcanic rocks based on the Total Alkali-Silica diagram, J. Petrol., 27, 745-750, 1986.

Lézine, A. M., von Grafenstein, U., Andersen, N., Belmecheri, S., Bordon, A., Caron, B., Cazet, J. P., Erlenkeuser, H., Fouache, E., Grenier, C., Huntsman-Mapila, P., Hureau-Mazaudier, D., Manelli, D., Mazaud, A., Robert, C., Sulpizio, R., Tiercelin, J. J., Zanchetta, G., and Zeqollari, Z.: Lake Ohrid, Albania, provides an exceptional multi-proxy record of environmental changes during the last glacial-interglacial cycle, Palaeogeogr. Palaeocl., 287, 116-127, 2010.

Lindhorst, K., Vogel, H., Krastel, S., Wagner, B., Hilgers, A., Zander, A., Schwenk, T., Wessels, M., and Daut, G.: Stratigraphic analysis of lake level fluctuations in Lake Ohrid: an integration of high resolution hydro-acoustic data and sediment cores, Biogeosciences Discuss., 7, 3651-3689, doi:10.5194/bgd-7-3651-2010, 2010.

Lucchi, F., Tranne, C. A., De Astis, G., Keller, J., Losito, R., and 
Morche, W.: Stratigraphy and significance of Brown Tuffs on the Aeolian Islands (southern Italy), J. Volcanol. Geoth. Res., 177, 49-70, 2008.

Margari, V., Pyle, D. M., Bryant, C., and Gibbard, P. L.: Mediterranean tephra stratigraphy revisited: Results from a long terrestrial sequence on Lesvos Island, Greece, J. Volcanol. Geoth. Res., 163, 34-54. 2007.

Marianelli, P. and Sbrana, A.: Risultati di misure di standard di minerali e di vetri naturali in microanalisi a dispersione di energia, Atti Soc, Tosc. Nat. Mem., Serie A, 105, 57-63, 1998.

Matzinger, A., Spirkovski, Z., Patceva, S., and Wüest, A.: Sensitivity of ancient Lake Ohrid to local anthropogenic impacts and global warming, J. Great Lakes Res., 32, 158-179, 2006.

Narcisi, B. and Vezzoli, L.: Quaternary stratigraphy of distal tephra layers in the Mediterranean - an overview, Global Planet. Change, 21, 31-50, 1999.

Orsi, G., De Vita, S., and Di Vito, M. A.: The restless, resurgent Campi Flegrei nested caldera (Italy): constraints on its evolution and configuration, J. Volcanol. Geoth. Res., 74, 179-214, 1996.

Pappalardo, L., Civetta, L., D’Antonio, M., Deino, A., Di Vito, M. A., Orsi, G., Carandente, A., De Vita, S., Isaia, R., and Piochi, M.: Chemical and Sr-isotopical evolution of the Phlegraen magmatic system before the Campanian Ignimbrite and the Neapolitan Yellow Tuff eruptions, J. Volcanol. Geoth. Res., 91, 141-166, 1999.

Pappalardo, L., Civetta, L., De Vita, S., Di Vito, M. A., Orsi, G., Carandente, A., and Fisher, R. V.: Timing of magma extraction during the Campanian Ignimbrite eruption (Campi Flegrei caldera), J. Volcanol. Geoth. Res., 114, 479-497, 2002.

Paterne, M., Guichard, F., and Labeyrie, J.: Explosive activity of the south Italian volcanoes durig the past 80000 years as determined by marine tephrochronology, J. Volcanol. Geoth. Res., 34, 153172, 1988.

Paterne, M., Labeyrie, J., Guichard, F., Mazaud, A., and Maitre, F.: Fluctuations of the Campanian explosive volcanic activity (south Italy) during the past 190000 years as determined by marine tephrochronology, Earth Planet. Sc. Lett., 98, 166-174, 1990.

Paterne, M., Guichard, F., Duplessy, J. C., Siani, G., Sulpizio, R., and Labeyrie, J.: A 90000-200000 yrs marine tephra record of Italian volcanic activity in the Central Mediterranean Sea, J. Volcanol. Geoth. Res., 177, 187-196, 2008.

Poli, S., Chiesa, S., Gillot, P. Y., and Guichard, F.: Chemistry versus time in the volcanic complex of Ischia (Gulf of Naples, Italy): evidence of successive magmatic cycles, Contrib. Mineral. Petr., 95, 322-335, 1987.

Popovska, C. and Bonacci, O.: Basic data on the hydrology of Lakes Ohrid and Prespa, Hydrol. Process., 21, 658-664, 2007.

Pyle, D. M., Ricketts, G. D., Margari, V., van Andel, T. H., Sinitsyn, A. A., Praslov, N., and Lisitsyn, S.: Wide dispersal and deposition of distal tephra during the Pleistocene "Campanian Ignimbrite/Y5" eruption, Italy, Quat. Sci. Rev., 25, 2713-2728, 2006.

Rosi, M. and Sbrana, A. (Eds.): The Phlaegrean Fields, Quaderni de "La ricerca Scientifica" 114. Consiglio Nazionale delle Ricerche, Rome, 1987.

Sadori, L. and Narcisi, B.: The postglacial record of environmental history from Lago di Pergusa, Sicily, Holocene, 11, 655-670, 2001.

Santacroce, R. (Ed.): Somma-Vesuvius, Quaderni de la Ricerca Sci- entifica, CNR, 114. Progetto Finalizzato Geodinamica, Monografie Finali, 8. CNR, Rome, 1987.

Santacroce, R., Cioni, R., Marianelli, P., Sbrana, A., Sulpizio, R., Zanchetta, G., Donahue, D. J., and Joron, J. L.: Age and whole rock-glass compositions of proximal pyroclastics from the major explosive eruptions of Somma-Vesuvius: a review as a tool for distal tephrostratigraphy, J. Volcanol. Geoth. Res., 177, 1-18, 2008.

St. Seymour, K. and Christianis, K.: Correlation of a tephra layer in Western Greece with a late Pleistocene eruption in the Campanian province of Italy, Quat. Res., 43, 46-54, 1995.

St. Seymour, K., Christanis, K., Bouzinos, A., Papazisimou, S., Papatheodorou, G., Moran, E., and Denes, G.: Tephrostratigraphy and tephrochronology in the Philippi peat basin, Macedonia, Northern Hellas (Greece), Quat. Internat., 121, 53-65, 2004.

Sulpizio, R., van Welden, A., Caron, B., and Zanchetta, G.: The Holocene tephrostratigraphic record of Lake Shkodra (Albania and Montenegro), J. Quaternary Sci., 25, 633-650, 2010.

Ton-That, T., Singer, B., and Paterne, M.: ${ }^{40} \mathrm{Ar} /{ }^{39} \mathrm{Ar}$ dating of latest Pleistocene (41 ka) marine tephra in the Mediterranean Sea: implications for global climate records, Earth Planet. Sc. Lett., 184, 645-658, 2001.

Vezzoli, L.: Island of Ischia, CNR, Quaderni della Ricerca Scientifica, CNR, Italy, 114(10), 230 pp., 1988.

Vogel, H., Zanchetta, G., Sulpizio, R., Wagner, B., and Nowaczyk, N.: A tephrostratigraphic record for the last glacial-interglacial cycle from Lake Ohrid, Albania and Macedonia, J. Quaternary Sci., 25, 320-338, 2010a.

Vogel, H., Wagner, B., Zanchetta, G., Sulpizio, R., and Rosén, P.: A Paleoclimate record with tephrochronological age control fort he last glacial-interglacial cycle from Lake Ohrid, Albania and Macedonia, J. Paleolimnol., 44, 295-310, 2010 b.

Wagner, B., Sulpizio, R., Zanchetta, G., Wulf, S., Wessels, M., and Daut, G.: The last $40 \mathrm{ka}$ tephrostratigraphic record of Lake Ohrid, Albania and Macedonia: a very distal archive for ash dispersal from Italian volcanoes, J. Volcanol. Geoth. Res., 177, 71$80,2008$.

Wagner, B., Lotter, A. F., Nowaczyk, N., Reed, J. M., Schwalb, A., Sulpizio, R., Valsecchi, V., Wessels, M., and Zanchetta, G.: A 40000-year record of environmental change from ancient Lake Ohrid (Albania and Macedonia), J. Paleolimnol., 41, 407-430, 2009.

Wagner, B., Vogel, H., Zanchetta, G., and Sulpizio, R.: Environmental changes on the Balkans recorded in the sediments from lakes Prespa and Ohrid, Biogeosciences Discuss., 7, 3365-3392, doi:10.5194/bgd-7-3365-2010, 2010.

Wulf, S., Kraml, M., Brauer, A., Keller, J., and Negendank, J. F. W.: Tephrochronology of the $100 \mathrm{ka}$ lacustrine sediment record of Lago Grande di Monticchio (southern Italy), Quat. Internat., 122, 7-30, 2004.

Zanchetta, G., Sulpizio, R., Giaccio, B., Siani, G., Paterne, M., Wulf, S., and D'Orazio, M.: The Y-3 Tephra: a Last Glacial stratigraphic marker for the central Mediterranean basin, J. Volcanol. Geoth. Res., 177, 145-154, 2008.

Zanchetta, G., Sulpizio, R., Roberts, N., Cioni, R., Eastwood, W. J., Siani, G., Paterne, M., and Santacroce, R.: Tephrostratigraphy, chronology and climatic events of the Mediterranean basin during the Holocene: an overview, The Holocene, doi:10.1177/0959683610377531, in press, 2010. 